\title{
Distinguishing Sichuan Walnut Cultivars and Examining Their Relationships with Juglans regia and J. sigillata by FISH, Early-Fruiting Gene Analysis, and SSR Analysis
}

\author{
Xiaomei Luo* and Jingyuan Chen \\ College of Forestry, Sichuan Agricultural University, Chengdu City, China
}

OPEN ACCESS

Edited by:

Jen-Tsung Chen,

National University of Kaohsiung,

Taiwan

Reviewed by:

Sezai Ercisli,

Atatürk University, Turkey

Sergio Mapelli,

Istituto di Biologia e Biotecnologia

Agraria (IBBA), Italy

*Correspondence:

Xiaomei Luo

xiaomei_luo@sicau.edu.cn

Specialty section:

This article was submitted to

Plant Breeding,

a section of the journal

Frontiers in Plant Science

Received: 18 October 2019

Accepted: 13 January 2020

Published: 25 February 2020

Citation:

Luo X and Chen J (2020) Distinguishing Sichuan Walnut Cultivars and Examining Their Relationships with Juglans regia and $\mathrm{J}$. sigillata by FISH, Early-Fruiting Gene Analysis, and SSR Analysis.

Front. Plant Sci. 11:27. doi: 10.3389/fp/s.2020.00027
Walnuts are economically important tree species in Sichuan Province (China) that provide heathy nuts. Fluorescence in situ hybridization (FISH) and analyses of an early-fruiting gene fragment and simple sequence repeats (SSRs) were used to distinguish Sichuan walnut cultivars and examine their relationships with Juglans regia L. and Juglans sigillata Dode. Thirty-four small chromosomes were counted in four Sichuan walnut cultivars. In the four cultivars, 5S rDNA was located in the proximal regions of two chromosomes (5 and 6), while $\left(\mathrm{AG}_{3} \mathrm{~T}_{3}\right)_{3}$ was located at both ends of each chromosome. The existence of the signal at both chromosome ends ensured accurate chromosome counts. 5S rDNA and $\left(\mathrm{AG}_{3} \mathrm{~T}_{3}\right)_{3}$ were not effective in identifying Sichuan walnut cultivars. Evolutionary analysis involving 32 early-fruiting nucleotide sequences from Sichuan walnut materials were performed with the maximum likelihood method. There were a total of 602 positions. All positions with gaps and missing data were eliminated, resulting in a final dataset of 562 positions. The ML tree with the highest log likelihood (-1607.82) revealed two obvious groups: one including materials of $\mathrm{J}$. regia, which fruits 1 year after grafting, and another including materials of $\mathrm{J}$. sigillata, which fruits $>3$ years after grafting. The early-fruiting gene fragment divided 22 walnut materials (10 walnut cultivars and 12 walnut accessions) into two groups, indicating that it was somewhat effective for distinguishing Sichuan walnut cultivars. Furthermore, 22 SSR loci were revealed to identify nine walnut cultivars. Eight cultivars were exclusively discerned by one SSR locus each: Chuanzao 1 [CUJRB307 (116) or CUJRA206a (182)], Chuanzao 2 [JSI-73 (154)], Shuangzao [CUJRB103a (123), CUJRB218 (144), JSI-71 (146), or CUJRA206a (176)], Shimianju [ZMZ11 (138)], Meigupao [CUJRB218 (149), CUJRB103a (151), or CUJRA206a (190)], Muzhilinhe [CUJRB220 (136), ZMZ11 (147), CUJRC310 (156), or JSI-73 (166)], Maerkang [CUJRA124 (154), CUJRB218 (159), or CUJRA123 (182)], Yanyuanzao [CUJRA124 (150) or CUJRA206a (192)]. The Shuling cultivar was identified by the combination of 
ZMZ11 (148) and other SSR loci, which distinguished and excluded the Chuanzao 1 and Yanyuanzao cultivars. Our results will guide the identification and breeding of Sichuan walnut cultivars.

Keywords: microsatellite marker, karyotype analysis, natural hybrids, artificial hybrids, walnuts

\section{INTRODUCTION}

Fruits are genetically very diverse groups grown in temperate, subtropical, and tropical regions and have been recognized for their human health benefits. Most of the fruits have high content of non-nutritive, nutritive, and bioactive compounds such as flavonoids, phenolics, anthocyanins, phenolic acids, and as well as nutritive compounds such as sugars, essential oils, carotenoids, vitamins, and minerals (Halasz et al., 2010; Alibabic et al., 2018; Butiuc et al., 2019). The species Juglans regia L. and Juglans sigillata Dode belong to Juglans in Juglandaceae, which consists of perennial woody plants that produce healthy nuts with a unique flavor and nutrient-rich composition (Kim et al., 2014). Sichuan Province is located at the boundary which is adjacent to the natural distributions of northern and southern walnuts in China. Northern walnuts (north of the Nibashan-Qinling Mountains), which grow under cold, dry, and high-sunshine conditions, and southern walnuts (south of the Erlangshan-Nibashan-HuangmaogengWumengshan Mountains), which grows under warm, dry, and low-sunshine conditions (Xi and Zhang, 1996), have been introduced to Sichuan. In this area, the introduced cultivars are exposed to high-temperature, high-humidity, and lowsunshine stresses, resulting in weak trees, severe fruit drop or fruitlessness. Northern walnut is classified as J. regia, while southern walnut is classified as J. sigillata. Sichuan walnut is highly likely to be a hybrid of these two varieties. The shapes of the nuts and compound leaves of these types of walnuts are shown in Figure 1. J. sigillata has more leaflets ( $\sim 7$ pairs) than J. regia ( 2 pairs), and the hybrids usually have $\sim 4$ pairs of leaflets. The compound leaf of J. sigillata contains a small, double parietal lobe (Figure 1A), while that of J. regia and the hybrids commonly has a large, single parietal lobe (Figures 1B, C). The walnut shell of J. sigillata has a deep texture (Figure 1D), while that of $J$. regia has little texture or may even be smooth (Figure 1E), and the shell of their hybrids has an intermediate texture (Figure 1F). Walnut cultivars vary greatly in quality (Pei and Lu, 2011), and tools for distinguishing among them are lacking. The engraftment scions (Chuanzao 1, Shuling, Chuanzao 2, Shuangzao, and Zaofeng) and the stock plants lack distinct characters (Figure 2). Therefore, a method to easily and accurately identify Sichuan walnut cultivars is greatly needed; such a method would also benefit breeders and planters. Walnut cultivars are usually distinguished based on morphology (Zenelli et al., 2005), supplemented by cytology (Tulecke et al., 1988), isozyme analysis (Fornari et al., 2001), restriction fragmentlength polymorphism (RFLP) analysis (Fjellstrom and Parfitt, 1994), randomly amplified polymorphic DNA (RAPD) analysis (Ross-Davis et al., 2008), inter-simple sequence repeat (ISSR) analysis (Christopoulos et al., 2010), amplified fragment-length polymorphism (AFLP) analysis (Mozaffarian et al., 2008), simple sequence repeat (SSR) analysis (Zhou et al., 2018a; Zhou et al., 2018b; Ebrahimi et al., 2019; Wu and Gmitter, 2019; Yan et al., 2019), single-nucleotide polymorphism (SNP) analysis (You et al., 2012), and high-throughput sequencing (Wu et al., 2019; Zhang et al., 2019; Zhu et al., 2019). However, morphological studies are preliminary breeding approaches, and morphological traits are usually quantitative, meaning that they are easily controlled by the environment and thus exhibit high variability (Andersen and Lübberstedt, 2003). In addition, similar characters cannot be distinguished by morphology (Zhao, 2007), and minor differences in tree cell structure cannot be detected by cytology (Luo et al., 2018). Furthermore, cytology is dependent on the experimental conditions and procedures used as well as on the methods used to prepare slides, with large differences among individual methods (Han et al., 2018). Elucidation of effective molecular markers for the identification of walnut cultivars requires sufficient genomic data. In addition, sample collection of late-fruiting walnut (which fruits after $>3$ years) is time consuming. Currently available methods are insufficient for the identification of Sichuan walnut cultivars; thus, there is a great need to establish an accurate and easy way to distinguish Sichuan walnut cultivars and to determine their relationships with J. regia and J. sigillata.

Chromosome number is a basic attribute used to establish the taxonomic statuses of walnut cultivars. Species in the genus Juglans have 32 chromosomes (Woodworth, 1930; Hans, 1970; Mu and Xi, 1988; Tulecke et al., 1988; Mu et al., 1990). At least five molecular genetic linkage maps for walnut have been established (Fjellstrom and Parfitt, 1994; Woeste et al., 1996; Malvolti et al., 2001; Luo et al., 2015; Zhu et al., 2015). Fluorescence in situ hybridization (FISH) with probe-labeled chromosome ends enables accurate counting of small walnut chromosomes (Deng et al., 2019; Liu and Luo, 2019; Luo and Liu, 2019a). Karyotype analysis aids in chromosome physical map construction and chromosome assembly (Hizume et al., 2002; Luo et al., 2017; Luo et al., 2018; Pereira et al., 2018), thereby providing a guide for distinguishing Sichuan walnut cultivars to a certain degree. However, no walnut cultivars have been subjected to FISH analysis. Furthermore, DNA sequencing is much more sensitive than FISH to species differences. Early fruiting, which is a dominant character controlled by at least two alleles, is an important attribute of walnut (Xi, 1987). Early fruiting walnut cultivars are those that fruit within three years after grafting. Sequence variation in early fruiting genes occurs in response to environmental stress. The harsh northern environment favours accelerated reproduction (Charrier et al., 2011); hence, earlyfruiting genes are commonly found in northern walnut (J. regia) 

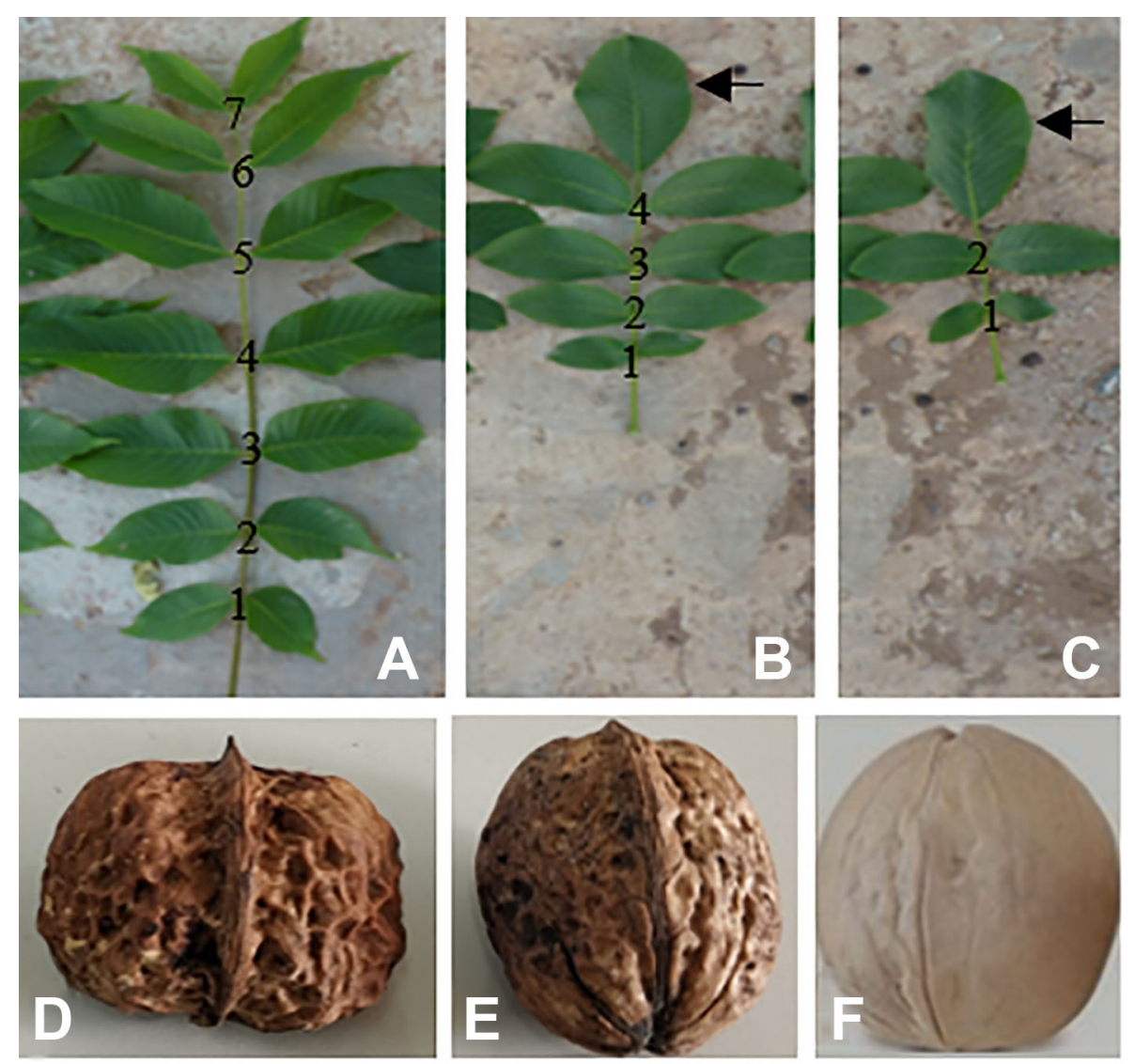

\section{Juglans sigillata \\ Hybrids Southern walnut Sichuan walnut Northern walnut}

FIGURE 1 | Morphological comparison of the leaves and nuts of J. sigillata, J. regia and their hybrids. J. sigillata has more leaflets (A, $\sim 7$ pairs) than J. regia (C, 2 pairs), and their hybrids usually have $\sim 4$ pairs of leaflets (B). The compound leaf of $J$. sigillata (A) has a small, double parietal lobe, while that of $J$. regia and the hybrids (B, C) commonly has a large, single parietal lobe (arrowed). The walnut shell of J. sigillata has a deep texture (D), while that of $J$. regia has little texture or may even be flat $\mathbf{( F )}$; the shells of the hybrids have intermediate textures $(\mathbf{E})$.

but seldom found in southern walnut (J. sigillata). Sichuan walnuts, as hybrids, have both early-fruiting ( $<3$ years) and late-fruiting ( $>3$ years) characteristics. Artificial hybrids, such as Chuanzao 1 (Pu et al., 2011) and Shuangzao (Xiao et al., 2013), and natural hybrids, such as Shimianju and Yanyuanzao (Sun et al., 2011) fruit 1 year after grafting. Natural hybrids, such as Meigupao and Muzhilinhe (Wang et al., 2017) do not fruit until $>3$ years after grafting or planting. Early-fruiting genes have high heritability (Ye et al., 2010). The SCAR marker linked to early bearing genes in the walnut was developed by Niu et al. (2008), and its terminal sequence was cloned by Zhu et al. (2011). The early-fruiting genes AGAMOUS, APETALA3, AP1, and LFAFY in J. regia were cloned and their expression analyzed by Breton et al. (2004), Ye and Niu (2011; 2012). However, no early-fruiting gene DNA sequences have been identified to distinguish Sichuan walnut cultivars. Analysis of SSRs, which are repeated units of 1 to 6 nucleotides, is a rapid, accurate, and effective technique that has been widely and successfully used to distinguish numerous walnut cultivars. Molecular studies on SSRs began to increase in the late 2000s. In 2016, the number of SSR loci identified in Juglans reached 15,000 (Bernard et al., 2018a), and this number has continued to increase (Shi et al., 2016; Ebrahimi et al., 2017; Bernard et al., 2018b; Chen et al., 2018; Zhou et al., 2018a; Zhou et al., 2018b; Ebrahimi et al., 2019; Chen et al., 2019; Wu et al., 2019). Although SSRs have been widely used for walnut identification, Sichuan walnut cultivars have not been well distinguished.

A total of 22 Sichuan walnut materials were used in this study. The karyotypes of four cultivars were analyzed in detail by FISH. The early-fruiting gene nucleotide sequences of 22 walnut materials (10 walnut cultivars and 12 walnut accessions) were subjected to evolutionary analysis, and nine walnut cultivars were subjected to SSR analysis. This work aimed to distinguish Sichuan walnut cultivars and examine their relationships with J. regia and J. sigillata with the goal of providing a guide for the identification and breeding of Sichuan walnut cultivars. 


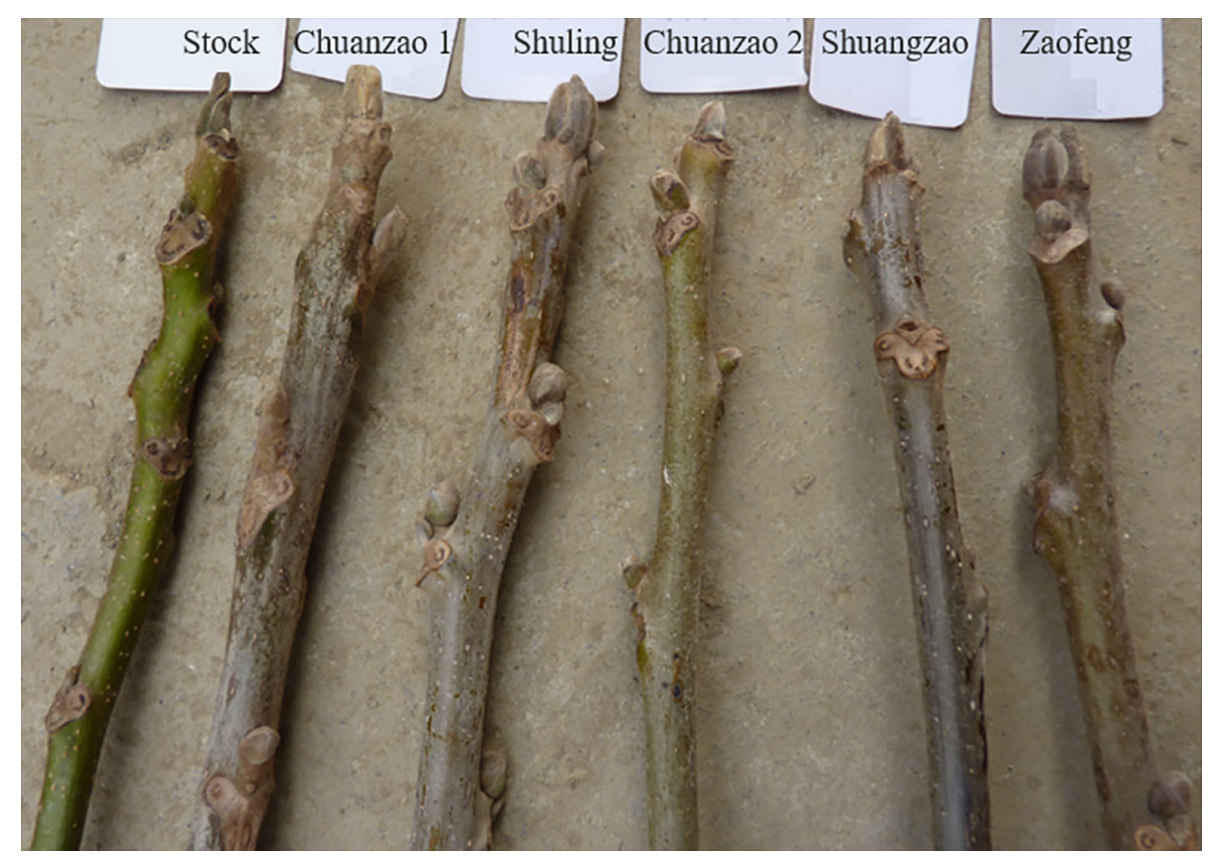

FIGURE 2 | Morphological comparison of engraftment scions of Chuanzao 1, Shuling, Chuanzao 2, Shuangzao, and Zaofeng and their stocks. No distinct characters were observed for any of the engraftment scions.

\section{MATERIALS AND METHODS}

In total, 10 walnut cultivars and 12 walnut accessions (belonging to J. regia or J. sigillata) were collected in Sichuan Province, China, for this study (Table 1). These materials included eight certified elite cultivars (Chuanzao 1, Chuanzao 2, Shuangzao, Shuling, Zaofeng, Shimianju, Muzhilinhe, and Yanyuanzao). Among these, there are five artificial hybrid cultivars, including (1) J. regia $\mathrm{L}$. 'Chuanzao 1 ' $=(J$. regia $\mathrm{L} . \times$ J. sigillata Dode 'Yunxin7926') $\times($ J. regia L. 'Xiazao') 'Chuanzao 1', (2) J. regia L. 'Chuanzao 2' $=($ J regia L. $\times$ J. sigillata Dode 'Yunxin7926' $) \times$ (J. regia L. 'Shahe') 'Chuanzao 2', (3) J. regia L. 'Shuangzao' $=(J$. regia L. $\times$ J. sigillata Dode 'Yunxin7926') $\times($ J. regia L. 'Xiazao') 'Shuangzao', (4) J. regia L. 'Shuling' $=($ J. regia L. $\times$ J. sigillata Dode 'Yunxin7926') $\times($ J. regia L. 'Xiazao') 'Shuling', and (5) J. regia L. 'Zaofeng' = (J. regia L. $\times$ J. sigillata Dode 'Yunxin7926') $\times($ J. regia L. 'Xiazao') 'Zaofeng'. The other seventeen walnut materials were likely natural hybrids.

\section{FISH Analysis}

Four Sichuan walnut cultivars, namely, Chuanzao 1, Muzhilinhe, Maerkang, and Yanyuanzao, were used in this experiment. Nuts from these cultivars were germinated in wet sand at room temperature. When the lateral roots reached a length of $\sim 2 \mathrm{~cm}$, their tips were cut off, treated with nitrous oxide, and stored in $75 \%$ alcohol. Chromosome slides were prepared by digesting meristems with cellulase and pectinase (2:1), stirring the meristems, and dropping the meristems in suspension onto slides. An Olympus CX23 microscope (Olympus, Japan) was used to examine the air-dried slides. A $5 \mathrm{~S}$ rDNA sequence
(41 bp) (Luo et al., 2017) and a chromosome-end repeat oligonucleotide $\left(\mathrm{AG}_{3} \mathrm{~T}_{3}\right)_{3}(21 \mathrm{bp}$ ) (Qi et al., 2002) were used for FISH analysis. The two probes were first tested in Juglans species. The oligonucleotide probes were synthesized by Sangon Biotech Co., Ltd. (Shanghai, China). The synthetic oligonucleotides were 5' end-labeled with 6-carboxyfluorescein (6-FAM) or 6carboxytetramethylrhodamine (6-TAMRA). The synthesized probes were diluted using a $1 \times \mathrm{TE}$ solution, maintained at a concentration of $10 \mu \mathrm{M}$ and then stored at $-20^{\circ} \mathrm{C}$.

Furthermore, well-spread slides were subjected to chromosome fixation, dehydration, and denaturation before being dehydrated again and then hybridized with the denatured probe mixture at $37^{\circ} \mathrm{C}$ for $2 \mathrm{~h}$. Subsequently, the chromosomes on the slides were washed, counterstained, and finally examined under an Olympus BX63 fluorescence microscope coupled to a Photometric SenSys Olympus DP70 CCD camera (Olympus, Japan). The raw images were examined in Photoshop version 7.1 (Adobe Systems Incorporated, San Jose, CA, USA). The chromosome nomenclature was based on length from the longest chromosome to the shortest chromosome. The chromosome ratio was calculated (over 3 metaphases) by dividing the length of the longest chromosome by the length of the shortest chromosome.

\section{Early-Fruiting Gene Analysis}

We examined 22 Sichuan walnut materials, including 10 cultivars and 12 accessions (Table 1). Total genomic DNA extraction was performed as described by Doyle and Doyle (1987), with a slight change. The early-fruiting gene was amplified with the forward primer 5'-TTTGTTGTTAGACTGAATGC-3' and the reverse 
TABLE 1 | The 22 materials used in this study, including 10 cultivars and 12 accessions.

\begin{tabular}{|c|c|c|c|}
\hline Walnut material & License number & Species & GenBank accession number \\
\hline Chuanzao 1_2 & Chuan S-SV-JSJR-001-2012 & Juglans regia L. 'Chuanzao 1' & MN548322 \\
\hline Shuangzao_1 & \multirow{2}{*}{ Chuan R-SC-JSJR-002-2009 } & \multirow{2}{*}{ Juglans regia L. 'Shuangzao' } & MN548308 \\
\hline Shuangzao_2 & & & MN548316 \\
\hline Shuling & Chuan R-SV-JSJR-003-2007 & Juglans regia L. 'Shuling' & MN548335 \\
\hline Zaofeng_1 & Chuan R-SC-SJR -008-2013 & Juglans regia L. 'Zaofeng' & MN548325 \\
\hline Muzhilinhe & Chuan R-SC-JR-002-2015 & Juglans sigillata Dode 'Muzhilinhe' & MN548332 \\
\hline Yanyuanzao_1 & \multirow{2}{*}{ Chuan S-SC-JR-003-2009 } & \multirow{2}{*}{ Juglans regia L. 'Yanyuanzao' } & MN548306 \\
\hline Yanyuanzao_2 & & & MN548309 \\
\hline Maerkang & - & Juglans sigillata Dode 'Maerkang' & MN548330 \\
\hline Meigupao & - & Juglans sigillata Dode 'Meigupao' & MN548331 \\
\hline 4 & - & Juglans sigillata Dode & MN548327 \\
\hline 10 & - & Juglans sigillata Dode & MN548337 \\
\hline $16 \_1$ & \multirow{3}{*}{-} & \multirow{3}{*}{ Juglans regia L. } & MN548315 \\
\hline 16_2 & & & MN548319 \\
\hline $16 \_3$ & & & MN548320 \\
\hline $20 \_1$ & \multirow[b]{2}{*}{-} & \multirow{2}{*}{ Juglans regia L. } & MN548312 \\
\hline 20_2 & & & MN548313 \\
\hline 32 & - & Juglans sigillata Dode & MN548334 \\
\hline 42 & - & Juglans regia $\mathrm{L}$. & MN548310 \\
\hline 45 & - & Juglans sigillata Dode & MN548321 \\
\hline $46 \_1$ & \multirow{4}{*}{-} & \multirow{2}{*}{ Juglans sigillata Dode } & MN548328 \\
\hline 46_2 & & & MN548336 \\
\hline $50 \_1$ & & & MN548323 \\
\hline 50_2 & & Juglans regia L. & MN548324 \\
\hline
\end{tabular}

primer 5'-GTGGATTTAAGGAAGGTTTG-3'. The primers were designed based on known early-fruiting sequences described by Ye et al. (2010). Each PCR $(50 \mu \mathrm{L})$ contained $15 \mu \mathrm{L}$ of polymerase mix, $1 \mathrm{mM}$ each primer, and $\sim 20 \mathrm{ng}$ of template DNA complemented with $\mathrm{ddH}_{2} \mathrm{O}$. The amplification protocols included 1 cycle of $5 \mathrm{~min}$ at $94^{\circ} \mathrm{C} ; 30$ cycles of $30 \mathrm{~s}$ at $94^{\circ} \mathrm{C}, 30 \mathrm{~s}$ at $62^{\circ} \mathrm{C}$, and $30 \mathrm{~s}$ at $72^{\circ} \mathrm{C}$; and $1 \mathrm{cycle}$ of $10 \mathrm{~min}$ at $72^{\circ} \mathrm{C}$. The PCR products were visualized on $0.8 \%$ agarose gels, purified with an ENZA $^{\mathrm{TM}}$ gel extraction kit (Omega, Georgia, USA), and then sequenced by the Beijing Genomics Institute (Beijing, China).

The sequences outside the primers were removed from the raw sequence data. The bidirectional sequences were matched in DNAMAN 6.0.3.99 (http://www.lynnon.com/). Thirteen materials had a single sequence, eight materials had two sequences, and one material had three sequences. Therefore, the 22 materials included 32 sequences, and these clean sequences were submitted to the National Center of Biotechnology Information (NCBI) and given GenBank accession numbers ranging from MN548306-MN548337 (Table 1). Subsequently, evolutionary analyses were conducted in MEGA X (Kumar et al., 2018). The evolutionary history of the early-fruiting gene was inferred by using the maximum likelihood method and the Tamura-Nei model (Tamura and Nei, 1993). The initial trees used for the heuristic search were obtained automatically by applying the Neighbor-Join and BioNJ algorithms to a matrix of pairwise distances estimated using the maximum composite likelihood (MCL) approach and then selecting the topology with the superior log likelihood value. The tree was drawn to scale, with the branch lengths indicating the number of substitutions per site. The codon positions included were $1 s t+2 n d+3 r d+$ noncoding.

\section{SSR Analysis}

Nine Sichuan walnut cultivars, namely, Chuanzao 1, Chuanzao 2, Shuangzao, Shuling, Shimianju, Meigupao, Muzhilinhe, Maerkang, and Yanyuanzao, were used for SSR analysis. Total genomic DNA extraction was performed as described by Doyle and Doyle (1987), with a slight change. Each PCR $(15 \mu \mathrm{L})$ contained $5 \mu \mathrm{L}$ of polymerase mix, $1 \mathrm{mM}$ each primer, and $\sim 20 \mathrm{ng}$ of template DNA complemented with $\mathrm{ddH}_{2} \mathrm{O}$. The amplification protocols included 1 cycle of $4 \mathrm{~min}$ at $95^{\circ} \mathrm{C} ; 25$ cycles (or 30 cycles depending on the primers) of $1 \mathrm{~min}$ at $94^{\circ} \mathrm{C}$, $1 \mathrm{~min}$ at $55^{\circ} \mathrm{C}$, and $1 \mathrm{~min}$ at $72^{\circ} \mathrm{C}$; and 1 cycle of $7 \mathrm{~min}$ at $72^{\circ} \mathrm{C}$. The forward primers were labeled with 6-FAM, HEX, and ROX.

Based on the genomic data for walnut (J. regia and J. sigillata) available from the NCBI (Martinez-Garcia et al., 2016; Luo and Liu, 2019b), 21 pairs of primers (Table 2) were obtained after two rounds of screening. Then, the fluorescent amplification products were sequenced on an ABI 3730 DNA analyser (Applied Biosystems), and allele sizing was performed using Gene Mapper ${ }^{\mathrm{TM}} 4.1$ software (Applied Biosystems). The obtained allele sizes and relative peak heights were analyzed in Excel 2019. 
TABLE 2 | The 21 SSR loci distinguish Sichuan walnut materials used in this study.

\begin{tabular}{|c|c|c|c|}
\hline SSR loci & Repeat motif & Product size (bp) & Forward $(F)$ and reverse $(R)$ primers (5'-3') \\
\hline CUJRA123 & $(\mathrm{AC})_{12}$ & 193 & F-TTGGTCTCTTCTITCCTCTATG R-TCGAACGTACAATAACGTACAG \\
\hline CUJRA124 & $(\mathrm{GT})_{13}$ & 151 & F-CGTTGCCTGAACAAGTAAGAT R-GAAGGAGGCTAACTCCCTATG \\
\hline CUJRA206a & $(\mathrm{AC})_{16}$ & 197 & F-GCCGAGAGAGGAAGAGAGACT R-CGACTACAGGGACCAATCAAC \\
\hline CUJRB012 & $(A G)_{15}$ & 102 & F-ACTCATCAAGATCCCCGACTAC R-CCACATCGTCTGGGTTCAT \\
\hline CUJRB103a & $(\mathrm{TC})_{17}$ & 148 & F-CATGCTATGGACTACCTCCTCR-AAGAGAGACGAACGAAGAGTG \\
\hline CUJRB218 & $(\mathrm{GA})_{13}$ & 159 & F-CTAGCGTCGAAGAAGAAGATG R-TTGTTTCTCCTCTGTCATGTाT \\
\hline CUJRB220 & $(\mathrm{TC})_{25}$ & 163 & F-AGCATGTATAGGCCAATGATG R-TCGTTCTATCTACAAGCACTCG \\
\hline CUJRB305 & $(\mathrm{GA})_{19}$ & 132 & F-GCTGCTITATAGCCATGATC R-GGTTCAATGTGCAACAAGAG \\
\hline CUJRB307 & $(\mathrm{GA})_{23}$ & 146 & F-CTGGGCTGAAGGAGAATC R-TTGGATGTCTGCПIITAGAG \\
\hline CUJRB317 & $(\mathrm{TC})_{17}$ & 125 & F-TGCCCACTAACCCTAACC R-GAGAAAAAGAATGGCTGTATTG \\
\hline CUJRC310 & $(\mathrm{TTG})_{7}$ & 142 & F-GCTGTTAGTGGAATCCCAACT R-TAAACGTGATCGAAGTGAAATG \\
\hline CUJRD204a & $(\mathrm{CTC})_{4}$ & 154 & F-CAGCCAATCTTCTTCTGCTTC R-GAGACCTACGACCACGATCAC \\
\hline $\mathrm{JH} 2753$ & $(\mathrm{GCT})_{6}$ & $188-212$ & F-CAGTITGGCCAGCTGCAAT R-TGTGCCCATGCTAAGACTGG \\
\hline JUG-13 & $(\mathrm{GGA})_{5}$ & $240-250$ & F-GAAGAGACTCCGTTGCCACA R-ACTCCGTCGTITCCCTGAAC \\
\hline JSI-15 & $(\mathrm{TC})_{6}$ & $180-200$ & F-ATGAGAGCCAGCCAACAGAC R-CGAGCGAGCAAGAGAGAGAG \\
\hline JSI-63 & $(\text { GATCG })_{5}$ & $180-200$ & F-TCCGGACAACTCCTCATCCT R-CTCTCCGCCGAGTCATGTAC \\
\hline JSI-71 & $(\mathrm{GCAGTA})_{8}$ & $135-155$ & F-AGCTAGCTCTCAAACAACAAGC R-ACAAACATGGCAACCTTCGTG \\
\hline JSI-73 & $(\mathrm{TGCTCG})_{5}$ & $160-175$ & F-AGCTCAACGGTCAAGGAAGG R-GGAGAGAGAGAGCTCGGCTA \\
\hline JRE-28 & $(\mathrm{AAG})_{5}$ & $150-170$ & F-CCGGGAAGCTCAGTTCAAGA R-GGTTCTTCCGCAGTTGGTCT \\
\hline JRE-46 & $(\mathrm{GAA})_{18}$ & $190-220$ & F-GCCTCTCCTCGTGCTCATIT R-ACTCGCTACTITCAGGCCC \\
\hline ZMZ11 & $(\mathrm{CTG})_{5}$ & 160 & F-CCAGAACCAGGAGCCAGCAA R-GACCATCGGCCCGAAAGTAA \\
\hline
\end{tabular}

\section{RESULTS}

\section{FISH Analysis}

The mitotic metaphase chromosomes of four walnut cultivars (Chuanzao 1, Muzhilinhe, Maerkang, and Yanyuanzao) after FISH are shown in Figure 3. Furthermore, the chromosomes shown in Figure 3 are presented individually in Figure 4. A total of 34 chromosomes were counted in all four walnut cultivars. $5 \mathrm{~S}$ rDNA oligonucleotides were located in the proximal regions of two chromosomes (5 and 6) in each cultivar (red), while $\left(\mathrm{AG}_{3} \mathrm{~T}_{3}\right)_{3}$ was located at both ends of each chromosome in each cultivar (green). The existence of a signal at both chromosome ends ensured accurate chromosome counts. The fluorescence intensity of $5 \mathrm{~S}$ rDNA was stronger than that of $\left(\mathrm{AG}_{3} \mathrm{~T}_{3}\right)_{3}$. The fluorescence intensity of $5 \mathrm{~S}$ rDNA varied, but that of $\left(\mathrm{AG}_{3} \mathrm{~T}_{3}\right)_{3}$ was consistent among the four cultivars. Among the cultivars, the Yanyuanzao cultivar exhibited the strongest fluorescence intensity of $5 \mathrm{~S}$ rDNA, the Maerkang cultivar exhibited the weakest fluorescence intensity, and the other two cultivars (Chuanzao 1 and Muzhilinhe) exhibited intermediate intensity. There were no number differences or obvious intensity differences in the fluorescence signals among the four cultivars. Since 5S rDNA and $\left(\mathrm{AG}_{3} \mathrm{~T}_{3}\right)_{3}$ were insufficient for identifying Sichuan walnut cultivars, further methods for detection were necessary.

The chromosomes of each cultivar were aligned by length in one column from longest (\#1) to shortest (\#34). The chromosome lengths of the Chuanzao 1, Muzhilinhe, Maerkang, and Yanyuanzao cultivars were 2.16 to $0.97 \mu \mathrm{m}$, 2.54 to $1.00 \mu \mathrm{m}, 2.65$ to $0.98 \mu \mathrm{m}$, and 2.13 to $0.98 \mu \mathrm{m}$, respectively. Since these lengths were less than $3 \mu \mathrm{m}$, the chromosomes of these four walnut cultivars are small chromosomes. The chromosomes of the Muzhilinhe and Maerkang cultivars were slightly larger than those of the other cultivars (Chuanzao 1 and Yanyuanzao). The chromosome ratios of the Chuanzao 1, Muzhilinhe, Maerkang, and Yanyuanzao cultivars were $2.23,2.54,2.70$, and 2.17 , respectively, indicating the following order in terms of chromosome asymmetry: Maerkang> Muzhilinhe> Chuanzao 1> Yanyuanzao. Due to the hidden centromere in a large proportion of the chromosomes, the long arm and short arm were not determined, and further karyotype analysis was not carried out. These limited karyotype data contributed little to the identification of the walnut cultivars.

\section{Early-Fruiting Gene Analysis}

To further distinguish Sichuan walnut cultivars, evolutionary analysis involving thirty-two early-fruiting nucleotide sequences from 22 walnut materials was performed with the maximum likelihood method; the results are shown in Figure 5. The tree with the highest log likelihood $(-1607.82)$ is shown. There were a total of 602 positions. All positions with gaps and missing data were eliminated, resulting in a final dataset of 562 positions. There were two obvious groups in the tree. The first group included nineteen early-fruiting nucleotide sequences covering eleven J. regia materials with fruiting one year after grafting. The second group included thirty early-fruiting nucleotide sequences covering the other eleven J. sigillata materials with fruiting $>3$ years after grafting or planting. The seven cultivars Chuanzao 1, Chuanzao 2, Yanyuanzao, Shuling, Zaofeng, Shuangzao, and Shimianju belonged to the first group, while the other three cultivars (Meigupao, Maerkang, and Muzhilinhe) belonged to the second group. Since the early-fruiting gene fragment divided the ten walnut cultivars into two groups, it was able to distinguish the cultivars to some degree, but further methods of detection were needed.

\section{SSR Analysis}

To effectively distinguish the walnut cultivars, we further developed 21 SSR loci to detect nine walnut cultivars. A scatter diagram of these 21 SSR loci is provided in Figure 6. The allele 

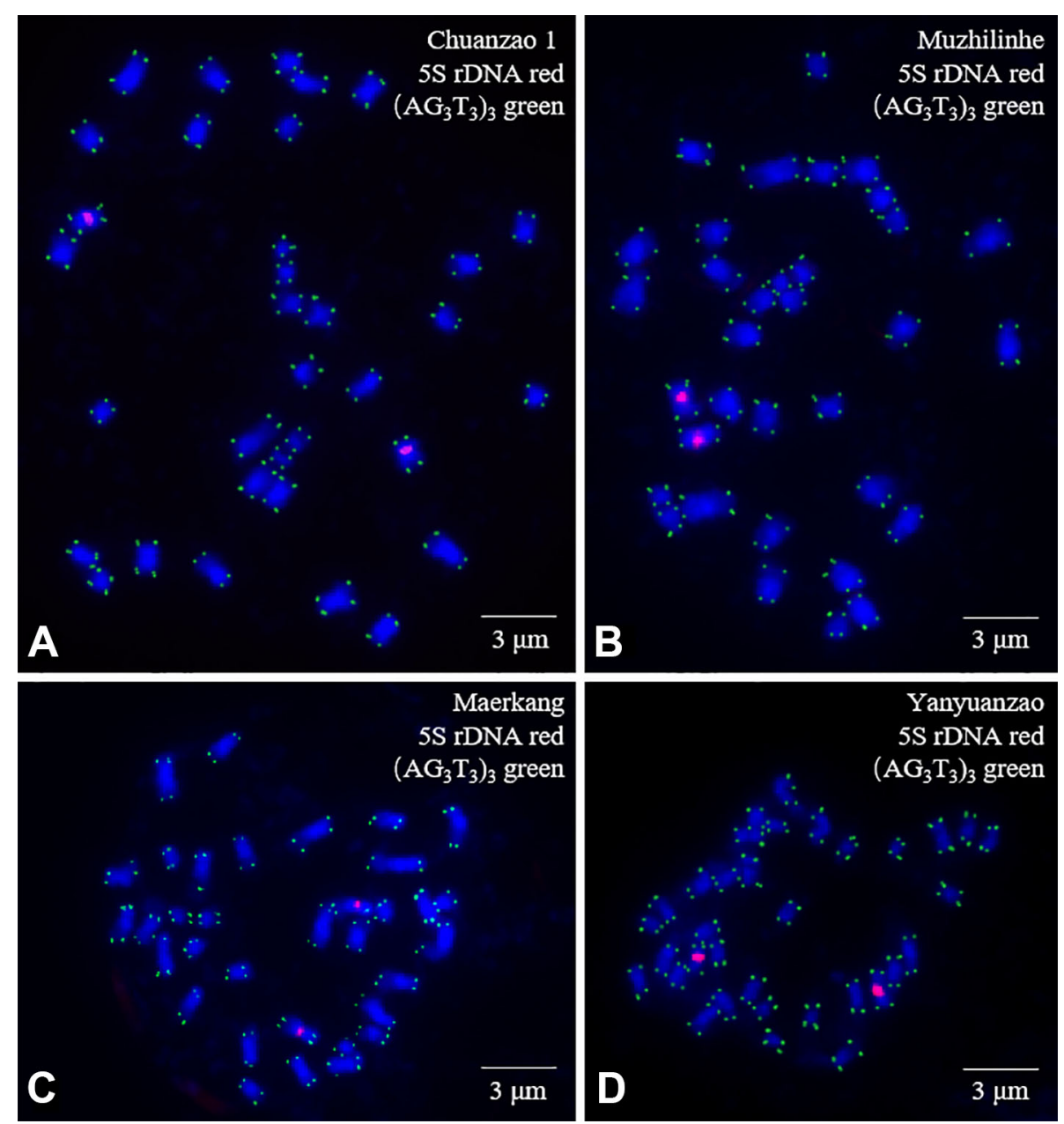

FIGURE 3 | Mitotic metaphase chromosomes of four walnut cultivars: (A) Chuanzao 1, (B) Muzhilinhe, (C) Maerkang, and (D) Yanyuanzao. Red represents 5S rDNA, while green represents $\left(\mathrm{AG}_{3} \mathrm{~T}_{3}\right)_{3}$. The blue chromosomes were counterstained by DAPI. Scale bar $=3 \mu \mathrm{m}$.

sizes ranged from 82 to 237 nucleotides, while the relative peak heights ranged from 201 to 32,722 . Most alleles were 135 to 200 nucleotides in length, while no alleles were 210 to 235 in size. Among the alleles, 3\% were less than 100 nucleotides, 9\% were greater than 200 nucleotides, and $87 \%$ were between 100 and 200 nucleotides in length. Half of the relative peak heights ranged from 25,000 to 32,722, while the others ranged from 200 to 25,000 . Among these relative peak heights, $21 \%$ were less than $10,000,15 \%$ were from 10,000 to $20,000,17 \%$ were from 20,000 to 30,000 , and $47 \%$ were greater than 30,000 .

Furthermore, the allele size of each of the 21 SSR loci is shown in Figure 7. In total, 183 allele sizes were obtained, excluding six missing allele sizes (white) for the Shuling cultivar. The SSR loci were aligned based on the allele size of the Chuanzao 1 cultivar from smallest (red) to largest (blue). To precisely and easily discern the four artificial hybrid cultivars and five natural hybrid cultivars, repeated allele sizes and relative peak heights less than 14,000 were filtered out. Subsequently, several effective allele sizes were maintained, as indicated by the purple ovals. Eleven
SSR loci helped discern eight of the cultivars (excluding the Shuling cultivar); these SSR loci were CUJRB307, CUJRA124, CUJRA123a, CUJRB220, ZMZ11, CUJRC310, JSI-71, JSI-73, CUJRB218, CUJRA206a, and CUJRA213. Each of the eight cultivars (Chuanzao 1, Chuanzao 2, Shuangzao, Shimianju, Meigupao, Muzhilinhe, Maerkang, and Yanyuanzao) was exclusively discerned by one SSR locus. Due to the lack of allele sizes, the Shuling cultivar was discerned by a repeat allele size of 148 nucleotides, as shown by the dashed oval. Hence, it was possible to discern the Shuling cultivar with a combination of ZMZ11 and other SSR loci, which distinguished and excluded the Chuanzao 1 and Yanyuanzao cultivars.

Figure 8 further shows the nine cultivars discerned by the 21 selected SSR allele sizes from eleven SSR loci summarized in Figure 7. The allele sizes ranged from 116 to 192 nucleotides, while the relative peak heights ranged from 14,643 to 32,607 . The Chuanzao 1 cultivar was exclusively discerned by CUJRB307 (116) or CUJRA206a (182); the Chuanzao 2 cultivar was exclusively discerned by JSI-73 (154); the Shuangzao cultivar 


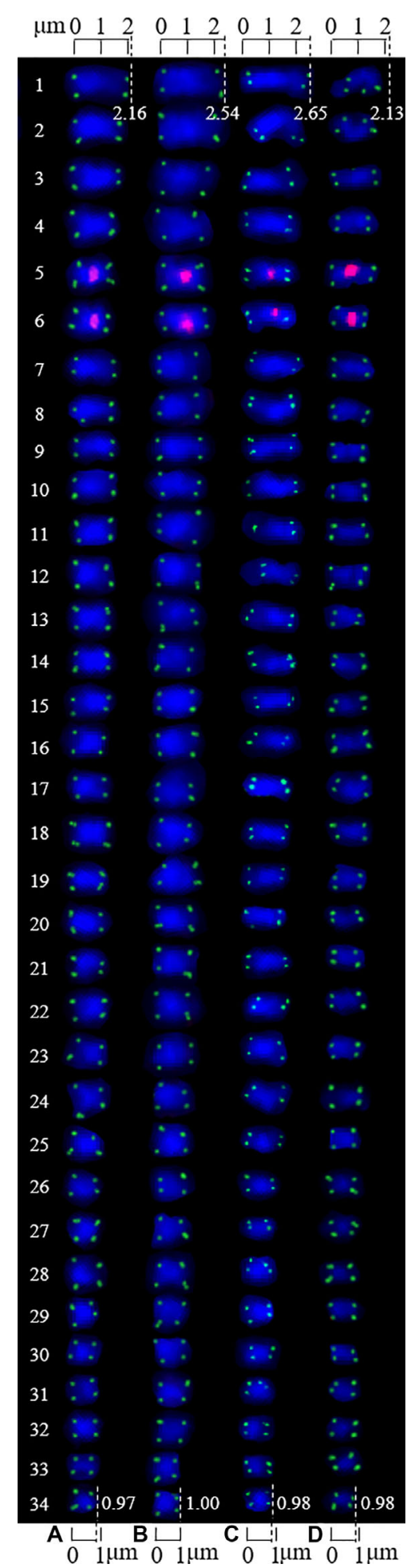

FIGURE 4 | Chromosomes from Figure $\mathbf{3}$ presented individually. The chromosomes were aligned by length from longest to shortest. The left number represents the chromosome number, while the top/bottom number represents the chromosome length. (A) Chuanzao 1, 2.16-0.97 $\mu \mathrm{m}$;

(B) Muzhilinhe, 2.54-1.00 $\mu \mathrm{m}$; (C) Maerkang, 2.65-0.98 $\mu \mathrm{m}$;

(D) Yanyuanzao, 2.13-0.98 $\mu \mathrm{m}$. was exclusively discerned by CUJRB103a (123), CUJRB218 (144), JSI-71 (146), or CUJRA206a (176); the Shimianju cultivar was exclusively discerned by ZMZ11 (138); the Meigupao cultivar was exclusively discerned by CUJRB218 (149), CUJRB103a (151), or CUJRA206a (190); the Muzhilinhe cultivar was exclusively discerned by CUJRB220 (136), ZMZ11 (147), CUJRC310 (156), or JSI-73 (166); the Maerkang cultivar was exclusively discerned by CUJRA124 (154), CUJRB218 (159), or CUJRA123 (182); the Yanyuanzao cultivar was exclusively discerned by CUJRA124 (150) or CUJRA206a (192); and the Shuling cultivar was discerned by the combination of ZMZ11 (148) and other SSR loci, which distinguished and excluded the Chuanzao 1 [CUJRB307 (116) or CUJRA206a (182)] and Yanyuanzao [CUJRA124 (150) or CUJRA206a (192)] cultivars.

\section{DISCUSSION}

Rapid and accurate identification of Sichuan walnut cultivars is particularly important in grafting propagated nut tree species both for practical breeding purposes and for cultivar proprietary rights protection. In this study, we used FISH, early-fruiting gene analysis and SSR analysis to identify Sichuan walnut cultivars. We will discuss the results with respect to the following two aspects: $i$ ) distinguishing Sichuan walnut cultivars by molecular cellular inheritance and $\mathrm{ii}$ ) determining the relationships of Sichuan walnuts with J. regia and J. sigillata.

\section{Distinguishing Sichuan Walnut Cultivars by Molecular Cellular Inheritance}

In contrast to morphological analysis, FISH, early-fruiting gene analysis and SSR analysis are three methods of molecular cellular inheritance analysis. The Sichuan walnut cultivars showed strong differences in morphological characters, such as leaf and nut shell characters. However, these characters are easily controlled by the environment. Therefore, we first performed FISH to distinguish Sichuan walnut cultivars. In contrast to the findings of previous studies, thirty-four chromosomes were detected by chromosome-end fluorescence signals in all four cultivars in this study. Several previous works have reported that Juglans has thirty-two chromosomes (Woodworth, 1930; Hans, 1970; Tulecke et al., 1988; Mu and Xi, 1988; Mu et al., 1990). The original chromosome images obtained by Hans (1970); Tulecke et al. (1988), and Woodworth (1930) have not been checked. However, thirty-four chromosomes were counted in the original image of J. regia (Figure 9A), but $\mathrm{Mu}$ and Xi (1988) reported thirty-two chromosomes. Similarly, thirty-four chromosomes were counted in the original image of J. sigillata (Figure 9B), whereas $\mathrm{Mu}$ et al. (1990) reported thirty-two chromosomes. A similar phenomenon has been observed in the Carya species $C$. aquatica $(2 \mathrm{n}=32)$, C. dabieshanensis $(2 \mathrm{n}=32)$, C. hunanensis $(2 \mathrm{n}=32)$, C. myristiciformis $(2 \mathrm{n}=32)$, C. tonkinensis $(2 \mathrm{n}=32)$, C. illinoinensis $(2 \mathrm{n}=34)$, C. ovata $(2 \mathrm{n}=34)$, C. floridana $(2 \mathrm{n}=$ $64)$, and C. texana $(2 \mathrm{n}=64)(\mathrm{Xu}, 2017)$. During adaptive evolution, the chromosome numbers and forms of several plants change via polyploidization, aneuploidization (caused by 


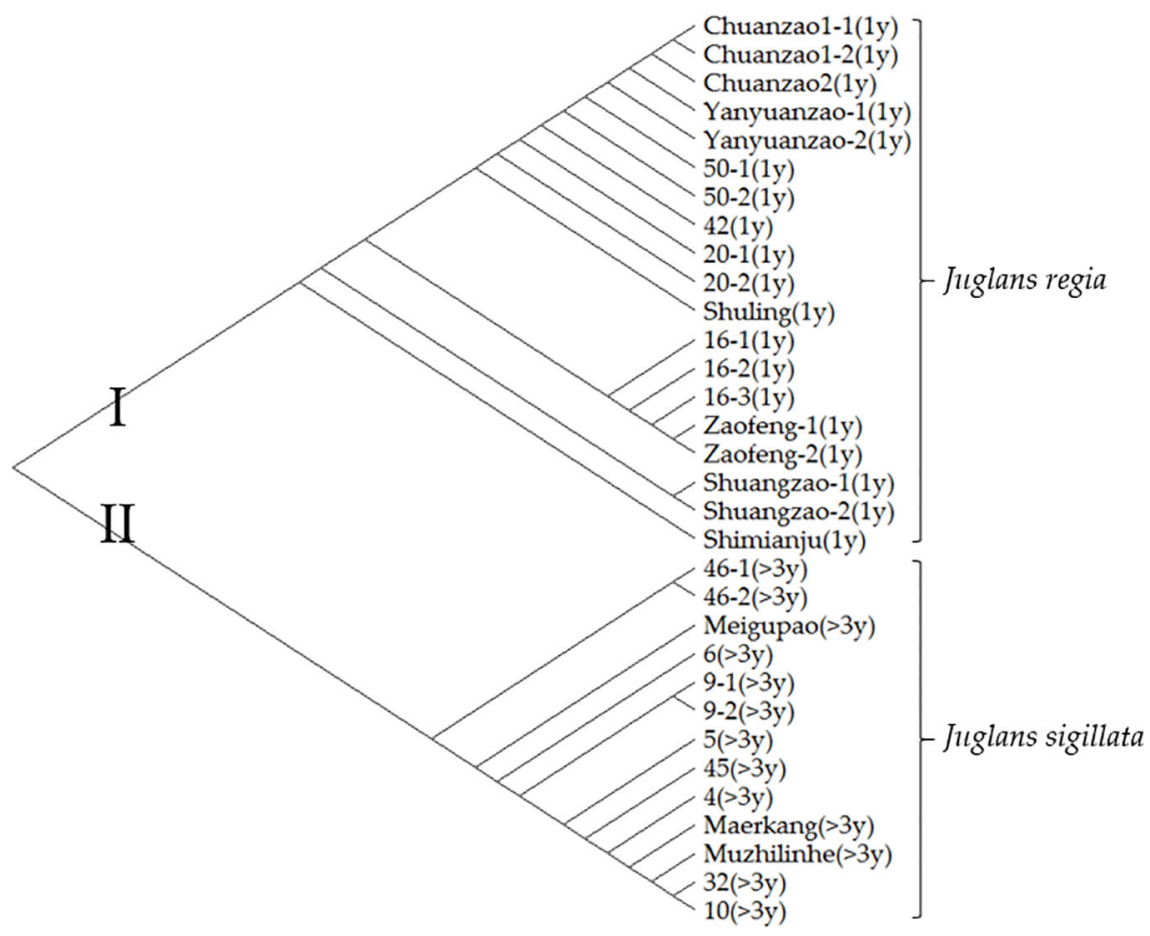

FIGURE 5 | Evolutionary analysis involving thirty-two early-fruiting nucleotide sequences from 22 walnut materials was performed with the maximum likelihood method. Evolutionary history was inferred by using the maximum likelihood method and the Tamura-Nei model. Evolutionary analyses were conducted in MEGA X. The information in parentheses represents the number of years after planting before fruiting occurs.

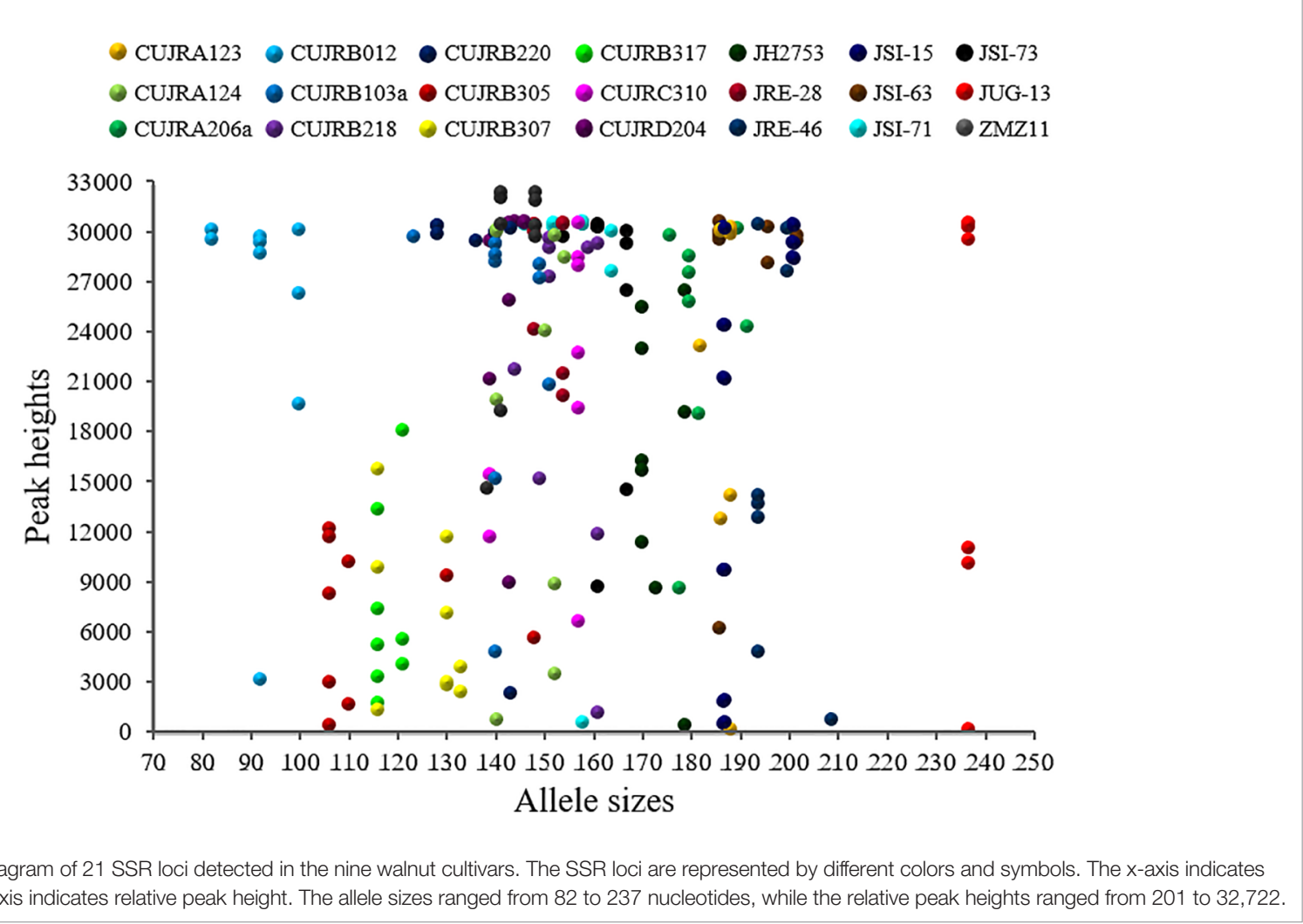




\begin{tabular}{|c|c|c|c|c|c|c|c|c|c|}
\hline \multirow[b]{2}{*}{ SSR loci } & \multicolumn{4}{|c|}{ Artificial hybrids } & \multicolumn{5}{|c|}{ Natural hybrids } \\
\hline & Chuanzaol & 1 Chuanzao2 & 2 Shuangzao & Shuling & Shimianju & Meigupao & Muzhilinhe & Maerkang & Yanyuanzao \\
\hline CUJRB012 & 92 & 92 & 100 & 92 & 82 & 100 & 92 & 82 & 100 \\
\hline CUJRB305 & 106 & 110 & 106 & 106 & 110 & 106 & 148 & 129 & 106 \\
\hline CUJRB307 & 116 & 129 & 135 & 117 & 129 & 129 & 117 & 129 & 135 \\
\hline CUJRB317 & 121 & 116 & 116 & - & 117 & 121 & 121 & 116 & 117 \\
\hline CUJRA124 & 140 & 152 & 140 & 140 & 152 & 152 & 140 & 154 & 150 \\
\hline CUJRB103a & 140 & 140 & 123 & 140 & 140 & 151 & 149 & 140 & 149 \\
\hline CUJRB220 & 143 & 141 & 128 & 143 & 128 & 141 & 136 & 128 & 141 \\
\hline CUJRD204a & 147 & 143 & 139 & - & 143 & 147 & 139 & 143 & 143 \\
\hline ZMZ11 & 148 & 141 & 141 & $14 \overline{8}$ & 138 & 141 & 147 & 141 & 148 \\
\hline JRE-28 & 154 & 154 & 154 & - & 148 & 154 & 147 & 148 & 147 \\
\hline CUJRC310 & 157 & 157 & 139 & - & 157 & 139 & 156 & 157 & 157 \\
\hline JSI-71 & 158 & 152 & 146 & 158 & 152 & 158 & 163 & 163 & 158 \\
\hline JSI-73 & 161 & 154 & 160 & 161 & 167 & 167 & 166 & 167 & 160 \\
\hline CUJRB218 & 161 & 151 & 144 & 161 & 161 & 149 & 151 & 159 & 151 \\
\hline $\mathrm{JH} 2753$ & 179 & 170 & 179 & 179 & 173 & 170 & 170 & 171 & 171 \\
\hline CUJRA206a & 182 & 180 & 176 & - & 178 & 190 & 180 & 180 & 192 \\
\hline JSI-15 & 187 & 187 & 201 & 187 & 201 & 187 & 201 & 187 & 187 \\
\hline CUJRA123 & 186 & 188 & 188 & 189 & 188 & 186 & 186 & 182 & 188 \\
\hline JSI-63 & 186 & 196 & 201 & 186 & 186 & 196 & 186 & 201 & 186 \\
\hline JRE-46 & 200 & 200 & 194 & - & 209 & 194 & 194 & 194 & 194 \\
\hline JUG-13 & 237 & 236 & 237 & 237 & 237 & 237 & 236 & 237 & 237 \\
\hline
\end{tabular}

FIGURE 7 | Allele sizes of 21 SSR loci of the four artificial hybrid cultivars and five natural hybrid cultivars. The SSR loci were aligned based on the allele size of the Chuanzao 1 cultivar from smallest to largest. Color gradients of red, blue, and green were used to mark all the allele sizes in Excel 2019 . Red represents small allele sizes, green represents large allele sizes, and light blue represents intermediate allele sizes. Six SSR loci of the Shuling cultivar were not obtained, as shown by "-" in the column for this cultivar. The allele sizes in purple ovals are those that can be used to discern the cultivars (except for allele size 148 in the dashed oval, because Shuling can be discerned by the combination of ZMZ11 (148) and other SSR loci, which distinguish and exclude Chuanzao 1 and Yanyuanzao.

deletion, duplication, inversion, or translocation), and $\mathrm{B}$ chromosome generation (Schubert, 2007; Guerra, 2008; Raskina et al., 2008; Weiss-Schneeweiss et al., 2008). For example, in plants in the genera Carex and Prospero, chromosomes commonly undergo breakage and fusion (Hipp and Roalson, 2009; Chung et al., 2011; Jang et al., 2013), especially Carex species, in which chromosome numbers range from 6 to 46 (Roalson, 2008). Both Trifolium subterraneum $(2 \mathrm{n}=$ 16) and Trifolium israeliticum $(2 \mathrm{n}=12)$ are produced by aneuploidization of $T$. israeliticum $(2 \mathrm{n}=14)$ (Falistocco et al., 2013). In Brassicaceae plants, a chromosome count of $n=8$ can be reduced to a count of $n=7$ (Mandakova and Lysak, 2008). The numbers of chromosomes vary among individuals within populations of Phaseolus vulgaris (Pedrosa-Harand et al., 2006). Hence, the possible reasons for the differences in walnut chromosome numbers between previous works and this study are as follows: $i$ ) Adaptive evolution. Aneuploidization variation in the four Sichuan walnut cultivars is likely because the chromosome numbers varied from 32 to 34 . ii) Amongpopulation variation. Similar to those of $P$. vulgaris, $C$. illinoinensis, and C. ovata, the chromosome numbers of the four Sichuan walnut cultivars probably vary among populations of individuals, and we determined the walnut cultivars to have $2 \mathrm{n}=34$. iii) Hybridization. Continuous hybridization has probably caused the chromosomes of the four Sichuan walnut cultivars to become mismatched and to misdivide during meiosis and mitosis because the parents have different genetic backgrounds. iv) Inaccurate chromosome number counts. Due to a lack of available technology for effectively discerning intact chromosomes, the chromosomes did not spread very well in previous study.

Although walnut chromosome numbers have been reported previously, the FISH technique has not yet been used to analyse walnut chromosomes. The FISH $5 S$ rDNA oligonucleotide with two signals and $\left(\mathrm{AG}_{3} \mathrm{~T}_{3}\right)_{3}$ with an end signal on every chromosome showed almost no differences among the four walnut cultivars in this study. Similar distribution patterns have also been observed in Citrus species (Deng et al., 2019); Berberis species (Liu and Luo, 2019); and Fraxinus, Syringa, and Ligustrum species (Luo and Liu, 2019a). FISH probes $\left(\mathrm{AG}_{3} \mathrm{~T}_{3}\right)_{3}$ located at chromosome ends ensure accurate chromosome counts (Pereira et al., 2018), although $\left(\mathrm{AG}_{3} \mathrm{~T}_{3}\right)_{3}$ is also occasionally observed in the internal positions of chromosomes (Murray et al., 2002; Majerová et al., 2014; Vasconcelos et al., 2018). Because 5S rDNA and $\left(\mathrm{AG}_{3} \mathrm{~T}_{3}\right)_{3}$ were inadequate for identifying the Sichuan walnut cultivars, it was necessary to perform further detection. The hidden centromeres of the small chromosomes hampered further karyotype analysis. A similar phenomenon has also been observed in Fraxinus pennsylvanica, Syringa oblata, Ligustrum lucidum, and Ligustrum $\times$ vicaryi (Luo and Liu, 2019a) as well as in Zanthoxylum armatum (Luo et al., 2018). The limited karyotype data contributed little to the identification of the walnut cultivars.

It is difficult for FISH to discern interspecific and intraspecific differences (Liu and Luo, 2019; Luo and Liu, 2019a). In addition, it is rather hard to prepare well-distributed chromosomes and 


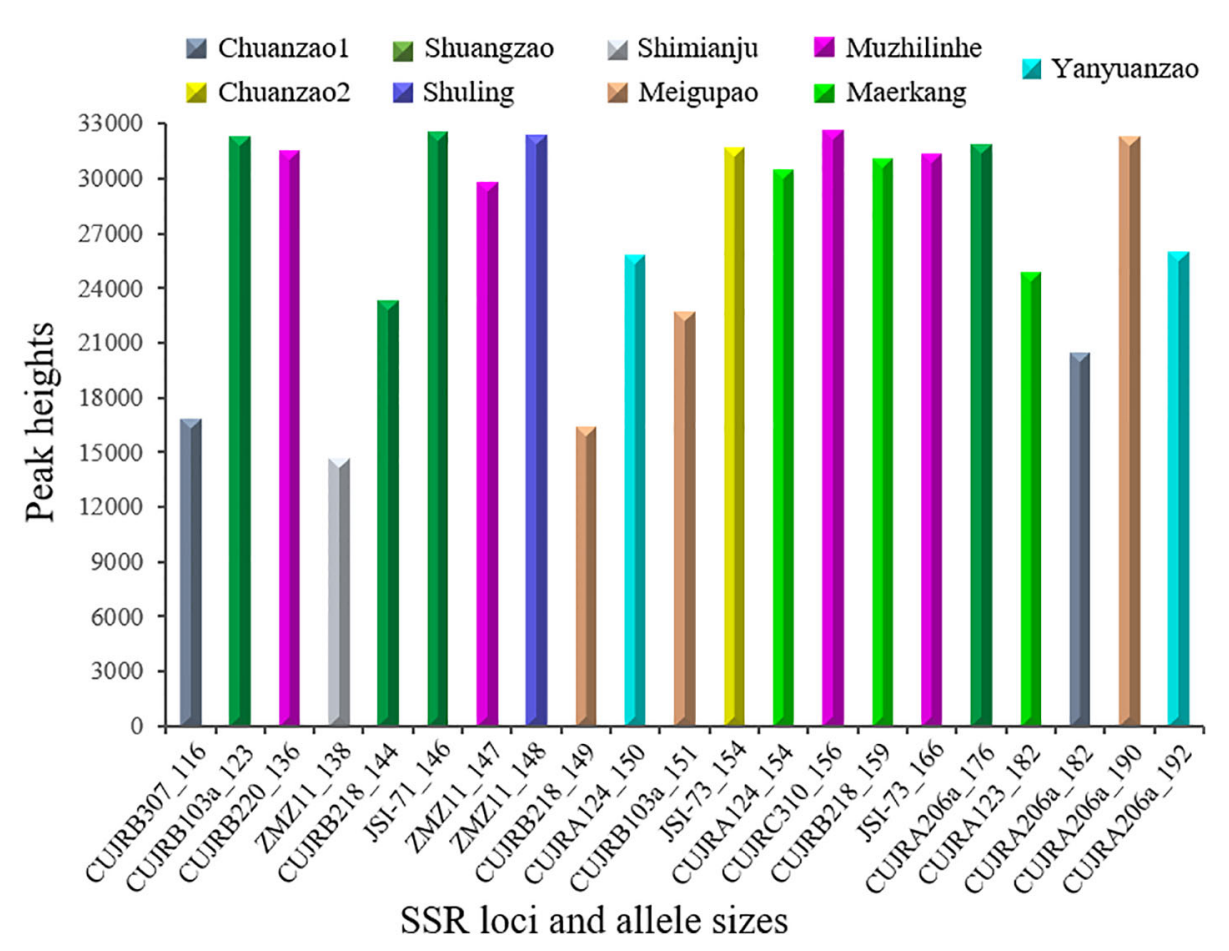

FIGURE 8 | Nine cultivars were discerned by 21 selected SSR allele sizes. The $x$-axis indicates SSR locus and allele size, while the $y$-axis indicates relative peak height. The allele size ranged from 116 to 192 nucleotides, while the relative peak height ranged from 14,643 to 32,607. The Chuanzao 1 cultivar was discerned by CUJRB307 (116) or CUJRA206a (182); Chuanzao 2 was discerned by JSI-73 (154); Shuangzao was discerned by CUJRB103a (123), CUJRB218 (144), JSI-71 (146), or CUJRA206a (176); Shimianju was discerned by ZMZ11 (138); Meigupao was discerned by CUJRB218 (149), CUJRB103a (151), or CUJRA206a (190); Muzhilinhe was discerned by CUJRB220 (136), ZMZ11 (147), CUJRC310 (156), or JSI-73 (166); Maerkang was discerned by CUJRA124 (154), CUJRB218 (159), or CUJRA123 (182); Yanyuanzao was discerned by CUJRA124 (150) or CUJRA206a (192); and Shuling was discerned by the combination of ZMZ11 (148) and other SSR loci, which distinguished and excluded Chuanzao 1 [CUJRB307 (116) or CUJRA206a (182)] and Yanyuanzao [CUJRA124 (150) or CUJRA206a (192)].

explore the ability of probes to identify walnut varieties. Root samples for chromosome counts should be collected from cuttings of sample trees. However, walnut plants rarely produce fat young roots; even seedlings usually grow all thin old roots. Hence, the root materials of the four walnut cultivars used in this study originated from germinated seeds. It takes approximately at least two months for walnuts to germinate, and then the lateral roots must branch from the main root. Root tips from lateral roots were used in this study. It was quite difficult to obtain even one wellspread slide. Furthermore, when potential sequences with differences between cultivars are obtained, these sequences must be converted into FISH probes. If these sequences are oligonucleotides ( $<60 \mathrm{bp}$ ), the FISH probes are inexpensive, easy to prepare, and effective. However, if these sequences are longer, preparation of FISH probes is expensive and more time consuming. When the probes are ready, additional slides are needed to test if the probes produce signals. In addition, for visualization of the fluorescence signal, the probes must be repeatedly used in adjacent chromosomes up to $10 \mathrm{kbp}$ long in metaphase (Pedersen and Linde-Laursen, 1995). Therefore, it is not easy to explore the identification ability of probes. In this study, we prepared wellspread chromosomes and developed probes located at chromosome ends and proximal regions, but the probes were not able to distinguish the walnut cultivars. Hence, it is necessary to explore more probes to identify walnut cultivars.

Furthermore, an evolutionary analysis of early-fruiting nucleotide sequences clearly revealed two groups of J. regia and J. sigillata in this study. DNA sequences of gene fragments can ordinarily be used to distinguish species in different genera; however, they are less well suited to reveal interspecies differences within the same genus and can rarely be used to discern cultivars in the same species. Previous works on earlyfruiting genes have mainly concentrated on J. regia and have included in vitro cultivation (Breton et al., 2004), molecular marker analysis (Niu et al., 2008; Ye et al., 2010), and cloning and expression analysis (Niu et al., 2008; Zhu et al., 2011). Few studies on early-fruiting genes in J. sigillata are available. The two species, J. regia and J. sigillata, have also been identified by restriction site-associated DNA SNPs (RAD-SNPs) (Yan et al., 2019) and RAPD (Chen et al., 2007). However, the chloroplast genomes of these two species differ little (Hu et al., 2017). In addition, early-maturing walnuts are more diverse than and are distinct from normally maturing walnuts (Ebrahimi et al., 2017). Walnuts feature precocious genotypes and exhibit a tendency towards homozygosity due to self-pollination (Chen et al., 2018). Since the early-fruiting gene fragment divided the ten walnut 

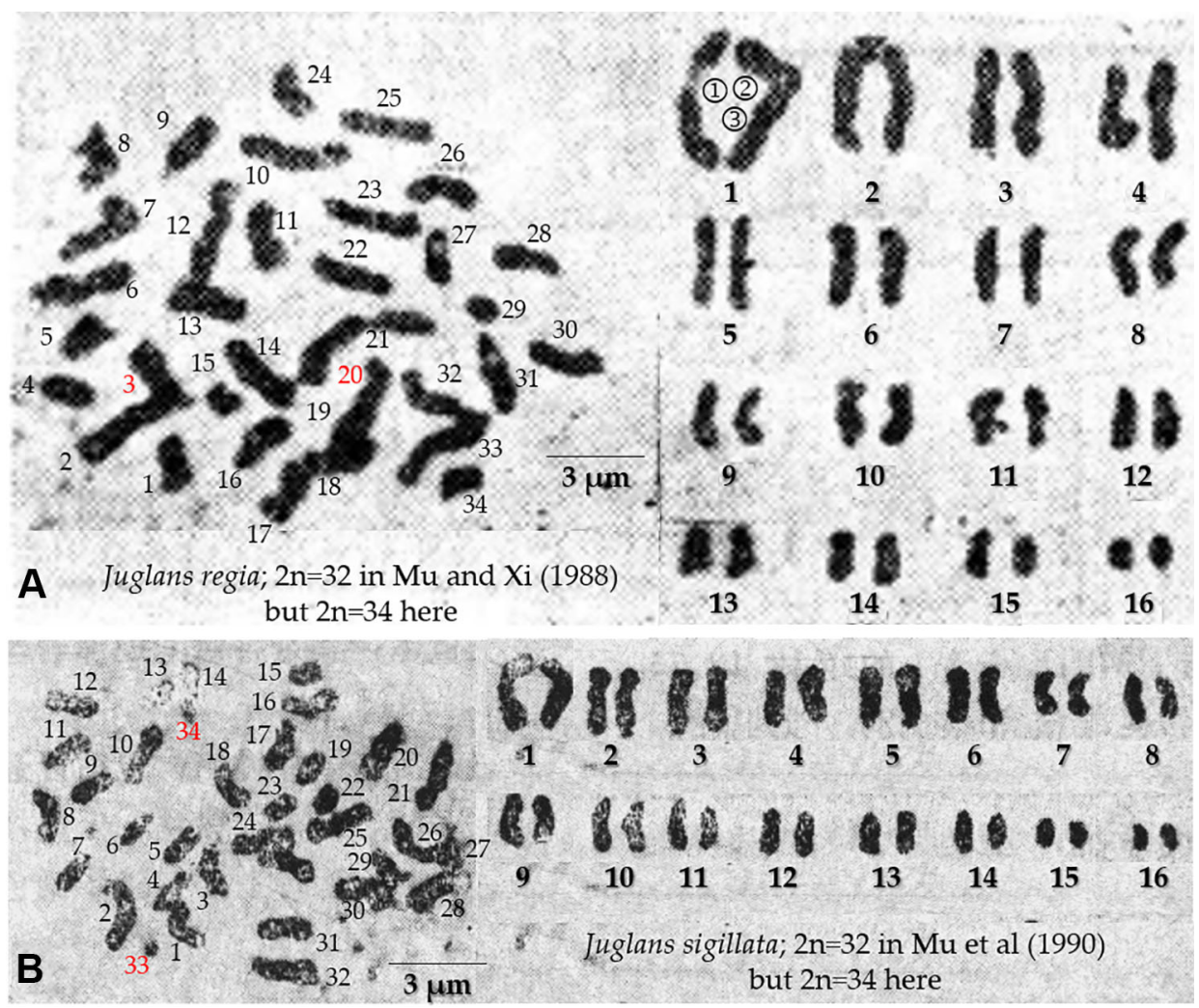

FIGURE 9 | Metaphase chromosomes of J. regia $\mathbf{( A )}$ and J. sigillata (B). Mu and Xi (1988) reported $2 \mathrm{n}=32 \mathrm{~J}$. regia chromosomes, but $2 \mathrm{n}=34$ were counted here. The first pair of chromosomes in (A) probably consists of three chromosomes, and the chromosomes with red numbers are likely disputed extra chromosomes. Mu et al. (1990) reported $2 n=32 \mathrm{~J}$. sigillata chromosomes, but $2 \mathrm{n}=34$ were counted here. The chromosomes with red numbers in (B) are likely disputed extra chromosomes.

cultivars into two groups, it was somewhat useful for recognition; however, additional methods of detection are still needed.

We further developed 21 SSR loci to detect nine Sichuan walnut cultivars in this study. Each of eight cultivars (Chuanzao 1, Chuanzao 2, Shuangzao, Shimianju, Meigupao, Muzhilinhe, Maerkang, and Yanyuanzao) was exclusively discerned by one SSR locus, and the Shuling cultivar was discerned by a combination of SSR loci. Shi et al. (2016) identified seven Sichuan walnut cultivars by selecting combinations of SSR loci from previous works (Woeste et al., 2002; Dangl et al., 2005; Ross and Woeste, 2008; Wang et al., 2011). Zhou et al. (2018a; 2018b) constructed SSR fingerprints of elite Sichuan cultivars of J. regia and J. sigillata. Although several walnut cultivars were examined by Shi et al. (2016) and Zhou et al. (2018a; 2018b), this study provided the following novel contributions: $i$ ) new SSR loci were developed, $i$ ) each of eight cultivars was exclusively discerned by one SSR locus, and iii) a few cultivars were tested for the first time. In total, approximately fifty elite walnut cultivars are licensed in Sichuan Province (http://www.sclmzm.com:88/sczmz/linmuliangzhong/ index.jhtml). To date, no study has distinguished all of these cultivars. The main barriers to doing so are difficulties in sample collection from all the cultivars and inadequate identification techniques. Hence, more work is needed to explore professional identification techniques for all the walnut cultivars in Sichuan. We are devoting to testing oligonucleotides probes from our SSR repeat motifs, developing more SSR loci that exclusively distinguish all the walnut cultivars in Sichuan, using additional methods such as Hi-C (High-throughput chromosome conformation capture) to construct chromosome conformation of Sichuan walnuts.

\section{Relationships of Sichuan Walnuts with J. regia and J. sigillata}

The geographical location of Sichuan has led to the development of natural hybrids of Sichuan walnuts, while social development and human demands have led to the production of artificial hybrids of Sichuan walnuts (Xiao and $\mathrm{Pu}, 2015$ ). It is unclear whether each hybrid Sichuan walnut belongs to one of its parent species (J. regia or $J$. sigillata) or represents a new taxon of Juglans. For example, the Shimianju cultivar was treated as J. regia by Sun et al. (2011); Shi et al. (2016), and Wu (2009) but as J. sigillata by Yan et al. (2019) and Zhou et al. (2018a; 2018b). Sichuan walnuts are intermediate between J. regia and J. sigillata in terms of their morphological characters (Xiao and $\mathrm{Pu}$, 2015; Zhou et al., 2018a; 2018b). Sichuan walnuts $(2 \mathrm{n}=34)$ have 
been reported to possess two more chromosomes than J. regia and J. sigillata in previous works (Woodworth, 1930; Hans, 1970; Tulecke et al., 1988; Mu and Xi, 1988; Mu and Xi, 1990). A probable reason for this increase in chromosome number is chromosome mismatching or misdivision during meiosis and mitosis caused by multiple hybridizations between J. regia and J. sigillata, which have genetic differences. Sichuan walnuts are different from J. regia and J. sigillata in terms of their morphological characters, and their chromosome numbers seem to provide some support for their classification in a new taxon of Juglans. However, the evolutionary analysis of the earlyfruiting gene of Sichuan walnut cultivars placed some of them within J. regia and some of them within J. sigillata, which does not support the grouping of Sichuan walnut cultivars in a new Juglans taxon. The SSR allele sizes of the Sichuan walnut cultivars were similar and did not support the existence of a new taxon of Juglans. In summary, Sichuan walnut cultivars vary greatly: some are similar to J. regia, some are similar to J. sigillata, and some are intermediate. However, these differences are not sufficient to treat these cultivars as a new Juglans taxon.

\section{DATA AVAILABILITY STATEMENT}

The DNA sequencing generated in this study was submitted to the National Center of Biotechnology Information (NCBI)

\section{REFERENCES}

Alibabic, A., Skender, A., Orascanin, M., Sertovic, E., and Bajric, E. (2018). Evaluation of morphological, chemical, and sensory characteristics of raspberry cultivars grown in Bosnia and Herzegovina. Turk. J. Agric. For. 42, 67-74. doi: 10.3906/tar-1702-59

Andersen, J. R., and Lübberstedt, T. (2003). Functional markers in plants. Trends Plant Sci. 8 (11), 554-560. doi: 10.1016/j.tplants.2003.09.010

Bernard, A., Lheureux, F., and Dirlewanger, E. (2018a). Walnut: past and future of genetic improvement. Tree Genet. Genomes 14, 1. doi: 10.1007/s11295-017-1214-0

Bernard, A., Barreneche, T., Lheureux, F., and Dirlewanger, E. (2018b). Analysis of genetic diversity and structure in a worldwide walnut (Juglans regia L.) germplasm using SSR markers. PloS One 13 (11), e0208021. doi: 10.1371/ journal.pone.0208021

Breton, C., Cornu, D., Chriqui, D., Sauvanet, A., Capelli, P., Germain, E., et al. (2004). Somatic embryogenesis, micropropagation and plant regeneration of "Early Mature" walnut trees (Juglans regia) that flower in vitro. Tree Physiol. 24 (4), 425-435. doi: 10.1093/treephys/24.4.425

Butiuc, A., Coste, A., Farkas, A., Cristea, V., Isac, V., and Halmagyi, A. (2019). Molecular characterization of apple (Malus $\times$ domestica Borkh.) genotypes originating from three complementary conservation strategies. Turk. J. Agric. For. 43, 464-477. doi: 10.3906/tar-1803-3

Charrier, G., Bonhomme, M., Lacointe, A., and Améglio, T. (2011). Are budburst dates, dormancy and cold acclimation in walnut trees (Juglans regia L.) under mainly genotypic or environmental control? Int. J. Biometeorol. 55, 763. doi: 10.1007/s00484-011-0470-1

Chen, L. H., Hu, T. X., and Zhang, F. (2007). Identification of relationship between Juglans regia L. and J. sigillata Dode by RAPD markers. J. Sichuan Agric. Univ. 25 (4), 513-516. doi: 10.16036/j.issn.1000-2650.2007.04.016

Chen, L., Dong, R., Ma, Q., Zhang, Y., Xu, S., Ning, D., et al. (2018). Precocious genotypes and homozygous tendency generated by self-pollination in walnut. BMC Plant Biol. 18 (1), 323. doi: 10.1186/s12870-018-1549-1

Chen, F., Chen, J., Wang, Z., Zhang, J., Li, X., Lin, M., et al. (2019). Genomics: cracking the mysteries of walnuts. J. Genet. 98 (2), 33. doi: 10.1007/s12041-0191084-3 and given GenBank accession numbers ranging from MN548306-MN548337.

\section{AUTHOR CONTRIBUTIONS}

Conceptualization: XL. Methodology: JC. Software: JC. Validation: XL. Formal analysis: XL. Investigation: JC. Resources: XL. Data curation: XL. Writing-original draft preparation: XL. Writing-review and editing: XL. Visualization: JC. Supervision: XL. Project administration: XL. Funding acquisition: XL. All authors consented to this submission.

\section{FUNDING}

This research was funded by the Natural Science Foundation of China (grant number 31500993).

\section{ACKNOWLEDGMENTS}

The authors thank Professor Zhou Yonghong for laboratory equipment support.

Christopoulos, M. V., Rouskas, D., Tsantili, E., and Bebeli, P. J. (2010). Germplasm diversity and genetic relationships among walnut (Juglans regia L.) cultivars and Greek local selections revealed by inter-simple sequence repeat (ISSR) markers. Sci. Hortic. 125 (4), 584-592. doi: 10.1016/j.scienta.2010.05.006

Chung, K. S., Weber, J. A., and Hipp, A. L. (2011). Dynamics of chromosome number and genome size variation in a cytogenetically variable sedge (Carex scoparia var. scoparia, Cyperaceae). Amer. J. Bot. 98 (1), 122-129. doi: 10.3732/ajb.1000046

Dangl, G. S., Woeste, K., Aradhya, M. K., Koehmstedt, A., Simon, C., Potter, D., et al. (2005). Characterization of 14 microsatellite markers for genetic analysis and cultivar identification of walnut. J. Amer. Soc Hortic. Sci. 130 (3), 348-354. doi: 10.21273/JASHS.130.3.348

Deng, H. H., Xiang, S. Q., Guo, Q. G., Jin, W. W., and Liang, G. L. (2019). Molecular cytogenetic analysis of genome-specific repetitive elements in Citrus clementina Hort. Ex Tan. and its taxonomic implications. BMC Plant Biol. 19, 77-88. doi: 10.1186/s12870-019-1676-3

Doyle, J., and Doyle, J. (1987). A rapid procedure for DNA purification from small quantities of fresh leaf tissue. Phytochem. Bull. 19, 11-15.

Ebrahimi, A., Zarei, A., Zamani, F. M., and Lawson, S. (2017). Evaluation of genetic variability among "Early Mature" Juglans regia using microsatellite markers and morphological traits. Peer J. 5, e3834. doi: 10.7717/peerj.3834

Ebrahimi, A., Mathur, S., Lawson, S. S., LaBonte, N. R., Lorch, A., Coggeshall, M. V., et al. (2019). Microsatellite borders and micro-sequence conservation in Juglans. Sci. Rep. 9 (1), 3748. doi: 10.1038/s41598-019-39793-z

Falistocco, E., Marconi, G., Falcinelli, M., and Scoles, G. J. (2013). Comparative cytogenetic study on Trifolium subterraneum $(2 \mathrm{n}=16)$ and Trifolium israeliticum $(2 \mathrm{n}=12)$. Genome 56 (6), 307-313. doi: 10.1139/gen-2013-0055

Fjellstrom, R. G., and Parfitt, D. E. (1994). RFLP inheritance and linkage in walnut. Theor. Appl. Genet. 89 (6), 665-670. doi: 10.1007/BF00223702

Fornari, B., Malvolti, M. E., Taurchini, D., Fineschi, S., Beritognolo, I., McCaglia, E., et al. (2001). Isozyme and organellar DNA analysis of genetic diversity in natural/naturalized European and Asiatic walnut (Juglans regia) populations. Acta Hortic. 544, 167-178. doi: 10.17660/ActaHortic.2001.544.23

Guerra, M. (2008). Chromosome numbers in plant cytotaxonomy: concepts and implications. Cytogenet. Genome Res. 120 (3-4), 339-350. doi: 10.1159/ 000121083 
Halasz, J., Pedryc, A., Ercisli, S., Yilmaz, K. U., and Hegedus, A. (2010). Sgenotyping supports the genetic relationships between Turkish and Hungarian apricot germplasm. J. Amer. Soc Horticult. Sci. 135 (5), 410-417. doi: 10.21273/ JASHS.135.5.410

Han, J., Kong, D. Y., and Peng, F. R. (2018). Optimization of chromosome mounting technique and karyotype analysis of Carya illinoinensis. Mol. Plant Breed. 16 (16), 5240-5246.

Hans, A. S. (1970). Chromosomal numbers in Juglandeceae. J. Arnold Abroretum 51, 534-539.

Hipp, A. L., and Roalson, R. E. H. (2009). Based on papers presented at two symposia on Cyperaceae held during the XVII international botanical congress in Vienna, Austria in July of 2005. The evolution of chromosome arrangements in Carex (Cyperaceae). Bot. Rev. 75 (1), 96-109. doi: 10.1007/s12229-008-9022-8

Hizume, M., Shibata, F., Matsusaki, Y., and Garajova, Z. (2002). Chromosome identification and comparative karyotypic analyses of four Pinus species. Theor. Appl. Genet. 105, 491-497. doi: 10.1007/s00122-002-0975-4

$\mathrm{Hu}$, Y., Woeste, K. E., and Zhao, P. (2017). Completion of the Chloroplast Genomes of Five Chinese Juglans and Their Contribution to Chloroplast Phylogeny. Front. Plant Sci. 7, 1955. doi: 10.3389/fpls.2016.01955

Jang, T. S., Emadzade, K., Parker, J., Temsch, E. M., Leitch, A. R., Speta, F., et al. (2013). Chromosomal diversification and karyotype evolution of diploids in the cytologically diverse genus Prospero (Hyacinthaceae). BMC Evol. Biol. 13 (1), 136. doi: 10.1186/1471-2148-13-136

Kim, H., Yokoyama, W., and Davis, P. A. (2014). TRAMP prostate tumor growth is slowed by walnut diets through altered IGF-1 levels, energy pathways, and cholesterol metabolism. J. Med. Food 17 (12), 1281-1286. doi: 10.1089/ jmf.2014.0061

Kumar, S., Stecher, G., Li, M., Knyaz, C., and Tamura, K. (2018). MEGA X: Molecular evolutionary genetics analysis across computing platforms. Mol. Biol. Evol. 35, 1547-1549. doi: 10.1093/molbev/msy096

Liu, J., and Luo, X. (2019). First report of bicolour FISH of Berberis diaphana and B. soulieana reveals interspecific differences and co-localization of (AGGGTTT) ${ }_{3}$ and rDNA 5S in B. diaphana. Hereditas 156, 13-21. doi: 10.1186/s41065-019-0088-6

Luo, X. M., and Liu, J. C. (2019a). Fluorescence in situ hybridization (FISH) analysis of the locations of the oligonucleotides $5 \mathrm{~S}$ rDNA, (AGGGTTT) ${ }_{3}$, and $(\mathrm{TTG})_{6}$ in three genera of Oleaceae and their phylogenetic framework. Genes 10, 375. doi: 10.3390/genes 10050375

Luo, X. M., and Liu, J. C. (2019b). Transcriptome analysis of acid-responsive genes and pathways involved in polyamine regulation in iron walnut. Genes 10, 605. doi: 10.3390/genes10080605

Luo, M. C., You, F. M., Li, P., Wang, J. R., Zhu, T., Dandekar, A. M., et al. (2015). Synteny analysis in Rosids with a walnut physical map reveals slow genome evolution in long-lived woody perennials. BMC Genomics 16 (1), 707. doi: 10.1186/s12864-015-1906-5

Luo, X. M., Liu, J. C., Zhao, A. J., Chen, X. H., Wan, W. L., and Chen, L. (2017). Karyotype analysis of Piptanthus concolor based on FISH with an oligonucleotide for rDNA 5S. Sci. Hortic. 226, 361-365. doi: 10.1016/j.scienta.2017.09.003

Luo, X. M., Liu, J. C., Wang, J. Y., Gong, W., Chen, L., and Wan, W. L. (2018). FISH analysis of Zanthoxylum armatum based on oligonucleotides for $5 \mathrm{~S}$ rDNA and (GAA) $)_{6}$. Genome 61, 699-702. doi: 10.1139/gen-2018-0009

Majerová, E., Mandáková, T., Vu, G. T., Fajkus, J., Lysak, M. A., and Fojtová, M. (2014). Chromatin features of plant telomeric sequences at terminal vs. internal positions. Front. Plant Sci. 5, 1-10. doi: 10.3389/fpls.2014.00593

Malvolti, M. E., Fornari, B., Maccaglia, E., and Cannata, F. (2001). Genetic linkage mapping in an intraspecific cross of walnut (Juglans regia L.) using molecular markers. Acta Hortic. 544, 179-185. doi: 10.17660/ActaHortic.2001.544.24

Mandakova, T., and Lysak, M. A. (2008). Chromosomal phylogeny and karyotype evolution in $\mathrm{x}=7$ Crucifer species (Brassicaceae). Plant Cell Online 20 (10), 2559-2570. doi: 10.1105/tpc.108.062166

Martinez-Garcia, P. J., Crepeau, M. W., Puiu, D., Gonzalezibeas, D., Whalen, J., Stevens, K., et al. (2016). The walnut (Juglans regia) genome sequence reveals diversity in genes coding for the biosynthesis of non-structural polyphenols. Plant J. 87 (5), 507-532. doi: 10.1111/tpj.13207

Mozaffarian, F., Mardi, M., Sarafrazi, A., and Ganbalani, G. N. (2008). Assessment of geographic and host-associated population variations of the carob moth, Ectomyelois ceratoniae, on pomegranate, fig, pistachio and walnut, using AFLP markers. J. Insect. Sci. 8, 6. doi: 10.1673/031.008.0601
Mu, Y. L., and Xi, R. T. (1988). Microsporogenesis studying and karyotype analysis of Juglans regia L. and J. hopeiensis Hu. J. Agric. Univ. Hebei 11 (4), 48-55. doi: CNKI:SUN:CULT.0.1988-04-007

Mu, Y. L., Xi, R. T., and Lv, Z. S. (1990). Microsporogenesis observation and karyotype analysis of some species in genus Juglans L. J. Wuhan Bot. Res. 8 (4), 301-310.

Murray, B. G., Friesen, N., and Heslop-Harrison, J. S. (2002). Molecular cytogenetic analysis of Podocarpus and comparison with other gymnosperm species. Ann. Bot. 89, 483-489. doi: 10.1093/aob/mcf047

Niu, J. X., Lv, J. Q., Wang, L., Ye, C. X., Zhang, H. P., and Zhang, L. X. (2008). SCAR marker linked to early-bearing genes in walnut. J. Fruit Sci. 25 (5), 732 735. doi: 10.13925/j.cnki.gsxb.2008.05.023

Pedersen, C., and Linde-Laursen, I. (1995). The relationship between physical and genetic distances at the Hor1 and Hor2 loci of barley estimated by two-color fluorescent in situ hybridization. Theor. Appl. Genet. 91, 941-946. doi: 10.1007/ BF00223904

Pedrosa-Harand, A., Souza de Almeida, C. C., Mosiolek, M., Blair, M. M., Schweizer, D., and Guerra, M. (2006). Extensive ribosomal DNA amplification during Andean common bean (Phaseolus vulgaris L.) evolution. Theor. Appl. Genet. 112 (5), 924-933. doi: 10.1007/s00122-0050196-8

Pei, D., and Lu, X. Z. (2011). Walnut germplasm resource in China (Beijing: Chinese Forestry Press).

Pereira, T. T. P., dos Reis, A. C. C. C., Cardoso, D. C., and Cristiano, M. P. (2018). Molecular phylogenetic reconstruction and localization of the (TTAGG) telomeric repeats in the chromosomes of Acromyrmex striatus (Roger, 1863) suggests a lower ancestral karyotype for leafcutter ants (Hymenoptera). Comp. Cytogen. 12, 13-21. doi: 10.3897/CompCytogen.v12i1.21799

Pu, G. L., Xiao, Q. W., and Zhou, L. Y. (2011). A new early-fruiting walnut cultivar 'Chuanzao 1'. Acta Hortic. Sin. 38 (10), 2025-2026.

Qi, Z. X., Zeng, H., Li, X. L., Chen, C. B., Song, W. Q., and Chen, R. Y. (2002). The molecular characterization of maize B chromosome specific AFLPs. Cell Res. 12, 63-68. doi: 10.1038/sj.cr.7290111

Raskina, O., Barber, J. C., Nevo, E., and Belyayev, A. (2008). Repetitive DNA and chromosomal rearrangements: speciation-related events in plant genomes. Cytogenet. Genome Res. 120 (3-4), 351-357. doi: 10.1159/000121084

Roalson, E. H. (2008). A Synopsis of chromosome number variation in the Cyperaceae. Bot. Rev. 74 (2), 209-393. doi: 10.1007/s12229-008-9011-y

Ross, D. A., and Woeste, K. E. (2008). Microsatellite markers for Juglans cinerea L. and their utility in other Juglandaceae species. Conserv. Genet. 9, 465-469. doi: 10.1007/s10592-007-9337-8

Ross-Davis, A., Huang, Z., McKenna, J., Ostry, M., and Woeste, K. (2008). Morphological and molecular methods to identify butternut (Juglans cinerea) and butternut hybrids: relevance to butternut conservation. Tree Physiol. 28 (7), 1127-1133. doi: 10.1093/treephys/28.7.1127

Schubert, I. (2007). Chromosome evolution. Current. Opin. Plant Biol. 10 (2), 109 115. doi: 10.1016/j.pbi.2007.01.001

Shi, L. H., Zhu, P., Wei, T., Zhang, L., Pu, G. L., He, W. C., et al. (2016). SSR screening and identification of walnut (Juglans regia) cultivars. J. Fruit Sci. 33 (7), 783-793. doi: 10.13925/j.cnki.gsxb.20150528

Sun, Q., Xiao, Q. W., Luo, Y. F., Zhang, Z. C., and Liao, Y. H. (2011). Research on the main economic characters of the giant walnut in Shimian. North. Hortic. 18, 15-18. doi: CNKI:SUN:BFYY.0.2011-18-006

Tamura, K., and Nei, M. (1993). Estimation of the number of nucleotide substitutions in the control region of mitochondrial DNA in humans and chimpanzees. Mol. Biol. Evol. 10, 512-526. doi: 10.1093/oxfordjournals.mol bev.a040023

Tulecke, W., McGranahan, G., and Ahmadi, H. (1988). Regeneration by somatic embryogenesis of triploid plants from endosperm of walnut, Juglans regia L. cv Manregian. Plant Cell Rep. 7 (5), 301-314. doi: 10.1007/BF00269923

Vasconcelos, E. V., Vasconcelos, S., Ribeiro, T., Benko-Iseppon, A. M., and Brasileiro-Vidal, A. C. (2018). Karyotype heterogeneity in Philodendron s.l. (Araceae) revealed by chromosome mapping of rDNA loci. PloS One 13, e0207318. doi: 10.1371/journal.pone.0207318

Wang, H. G., Gao, Y., Zhang, Z. H., Zhao, S. G., Xuan, L. C., and He, F. Q. (2011). Comparison of walnut pollen's shape, size and vitality. Chin. Agric. Sci. Bul. 27 (10), 98-101. 
Wang, J. Y., Gong, W., Wang, P., Yang, W. L., Luo, Y. G., and Zeng, Q. Y. (2017). A New mini-fruit and thin shell walnut cultivar 'MuzhiLinhe'. Acta Hortic. Sin. 44 (S2), 2649-2470. doi: 10.16420/j.issn.0513-353x.2017-0831

Weiss-Schneeweiss, H., Tremetsberger, K., Schneeweiss, G. M., Parker, J. S., and Stuessy, T. F. (2008). Karyotype diversification and evolution in diploid and polyploid south American Hypochaeris (Asteraceae) inferred from rDNA localization and genetic fingerprint Data. Ann. Bot. 101 (7), 909-918. doi: 10.1093/aob/mcn023

Woeste, K., McGranahan, G., and Bernatzky, R. (1996). The identification and characterization of a genetic marker linked to hypersensitivity to the cherry leafroll virus in walnut. Mol. Breed. 2, 261-266. doi: 10.1007/BF00564203

Woeste, K., Burns, R., Rhodes, O., and Michler, C. (2002). Thirty polymorphic nuclear microsatellite loci from black walnut. J. Hered. 93 (1), 58-60. doi: 10.1093/jhered/93.1.58

Woodworth, R. H. (1930). Meiosis of micro-sporogenesis in the Juglandaceae. Am. J. Bot. 17, 863-869. doi: 10.1002/j.1537-2197.1930.tb04927.x

Wu, G. A., and Gmitter, F. G. (2019). Novel assembly strategy cracks open the mysteries of walnut genome evolution. Hortic. Res. 6, 57. doi: 10.1038/s41438019-0143-5

Wu, T., Chen, S. Y., Ning, D. L., Xiao, L. J., He, N., Tang, C. Y., et al. (2019). Genetic diversity of walnut germplasm in Nujiang Prefecture based on SSR. J. Fujian Agric. For. Univ. (Nat. Sci. Edit.) 48 (02), 252-258. doi: 10.13323/ j.cnki.j.fafu(nat.sci.).2019.02.018

Wu, K. Z. (2009). Study on genetic diversity and correlation of hybrid parents and $\mathrm{F}_{1}$ in walnut (Chengdu, China: Sichuan Agri. Univ.).

Xi, R. P., and Zhang, Y. P. (1996). Fruit Flora in China. Walnut (Beijing: China Forestry Press), 1-50.

Xi, S. K. (1987). Germplasm resources of Juglans L. and Juglans regia L. Breeding. Sci. Silvae Sin. 23 (3), 342-350.

Xiao, Q. W., and Pu, G. L. (2015). Plant walnuts scientifically in Sichuan Province (Chengdu: Sichuan Sci. Technol. Press).

Xiao, Q. W., Xiao, Q. G., Zhou, L. Y., Pu, G. L., Wu, K. Z., Hu, T. X., et al. (2013). A new early-maturing and thin shell walnut cultivar 'Shuangzao'. Acta Hortic. Sin. 40 (1), 179-180. doi: CNKI:SUN:GNZY.0.2013-03-008

$\mathrm{Xu}, \mathrm{C} . \mathrm{M}$. (2017). Analysis of karyotype and genome type of the Carya species (Beijing, China: Chinese Acad. Forestry).

Yan, S. Y., Zhu, P., Gong, W., Wang, J. Y., Wu, K. Z., Wu, C. Y., et al. (2019). Studies on genetic diversity of Juglans cultivar germplasms in Sichuan based on RAD-SNPs analysis. J. Trop. Subtrop. Bot. 27 (1), 19-28. doi: CNKI:SUN: RYZB.0.2019-01-004

Ye, C. X., and Niu, J. X. (2011). Cloning and expression analysis of the Ap1 homolog gene in Juglans regia L. J. Shihezi Univ. (Natural Sci.) 29 (6), 679-682. doi: 10.3969/j.issn.1007-7383.2011.06.004

Ye, C. X., and Niu, J. X. (2012). Cloning and analysis of the LFAFY homologous gene from seeding walnut of Xinjiang Province. Acta Agricult. Borealioccidentalis Sin. 21 (11), 146-150. doi: 10.7606/j.issn.1004-1389.2012.11.027
Ye, C. X., Niu, J. X., Lv, J. Q., Wang, L., Li, R., and Zhang, H. P. (2010). Analysis of the molecular maker related to early-seeding in walnut female parent and $\mathrm{F}_{1}$ by SCAR. Mol. Plant Breed. 5, 971-975. doi: 10.3969/mpb.008.000971

You, F. M., Deal, K. R., Wang, J., Britton, M. T., Fass, J. N., Lin, D., et al. (2012). Genome-wide SNP discovery in walnut with an AGSNP pipeline updated for SNP discovery in allogamous organisms. BMC Genomics 13, 354. doi: 10.1186/ 1471-2164-13-354

Zenelli, G., Kola, H., and Dida, M. (2005). Phenotypic variation in native walnut populations of Northern Albania. Sci. Hortic. 105, 91-100. doi: 10.1016/ j.scienta.2004.11.003

Zhang, B. W., Lin, L. X., Li, N., Yan, P. C., Jiang, X. H., Woeste, K. E., et al. (2019). Phylogenomics reveals an ancient hybrid origin of the Persian walnut. Mol. Biol. Evol. pii, msz112. doi: 10.1093/molbev/msz112

Zhao, M. (2007). Research progress in the identification technology of fruit tree germplasm. J. Anhui Agric. Sci. 35 (18), 5414-5416. doi: 10.3969/j.issn.05176611.2007.18.039

Zhou, Y. B., Zhu, P., Gong, W., Wang, J. Y., Min, S. Y., and Wu, K. Z. (2018a). SSR fingerprint construction and genetic diversity analysis of elite Juglans regia cultivars in Sichuan. Acta Bot. Boreal. Occident. Sin. 38 (7), 1254-1261. doi: 10.7606/j.issn.1000-4025.2018.07.1254

Zhou, Y. B., Zhu, P., Gong, W., Wang, J. Y., Min, S. Y., Wu, K. Z., et al. (2018b). DNA fingerprinting establishment and clustering analysis of Juglans sigillata improved variety from southwestern Sichuan based on SSR markers. Mol. Plant Breed. 16 (17), 5683-5689. doi: 10.13271/j.mpb.016.005683

Zhu, T. D., Niu, J. X., Ye, C. X., Liu, N., and Zhang, F. P. (2011). Cloning of the terminal sequence of the SCAR marker (1-1500) that linked to early-bearing genes in walnut. Xinjiang Agricult. Sci. 48 (3), 393-398.

Zhu, Y., Yin, Y., Yang, K., Li, J., Sang, Y., Huang, L., et al. (2015). Construction of a high-density genetic map using specific length amplified fragment markers and identification of a quantitative trait locus for anthracnose resistance in walnut (Juglans regia L.). BMC Genomics 16 (1), 614. doi: 10.1186/s12864-015-1822-8

Zhu, T., Wang, L., You, F. M., Rodriguez, J. C., Deal, K. R., Chen, L., et al. (2019). Sequencing a Juglans regia $\times J$. microcarpa hybrid yields high-quality genome assemblies of parental species. Hortic. Res. 6, 55. doi: 10.1038/s41438-0190139-1

Conflict of Interest: The authors declare that the research was conducted in the absence of any commercial or financial relationships that could be construed as a potential conflict of interest.

Copyright (C) 2020 Luo and Chen. This is an open-access article distributed under the terms of the Creative Commons Attribution License (CC BY). The use, distribution or reproduction in other forums is permitted, provided the original author(s) and the copyright owner(s) are credited and that the original publication in this journal is cited, in accordance with accepted academic practice. No use, distribution or reproduction is permitted which does not comply with these terms. 